\title{
The Dynamic Stability and Nonlinear Resonance of a Flexible Connecting Rod: Continuous Parameter Model
}

\author{
SHANG-ROU HSIEH and STEVEN W. SHAW \\ Department of Mechanical Engineering and Applied Mechanics, The University of Michigan, Ann Arbor, \\ MI 48109-2125, U.S.A.
}

(Received: 6 April 1992; accepted: 25 August 1992)

\begin{abstract}
The transverse vibrations of a flexible connecting rod in an otherwise rigid slider-crank mechanism are considered. An analytical approach using the method of multiple scales is adopted and particular emphasis is placed on nonlinear effects which arise from finite deformations. Several nonlinear resonances and instabilities are investigated, and the influences of important system parameters on these resonances are examined in detail.
\end{abstract}

Key words: Slider-crank mechanism, nonlinear resonance, dynamic stability, method of multiple scales.

\section{Introduction}

High operating speeds, superior reliability and accurate performance are major characteristics of modern industrial machinery and commercial equipment. The traditional rigid body analysis which presumes low operating speeds becomes insufficient for describing the performance of such high speed machinery. A thorough understanding of the dynamic behavior of machine elements undergoing high speed operations is necessary in these situations. It is the purpose of this investigation to present an analytical approach for describing the dynamic response of an elastic mechanism element and provide a theoretical explanation for some of the dynamic phenomena observed in both numerical and experimental works [1-4]. One of the simplest and most common mechanisms, the slider-crank mechanism, is selected to demonstrate this approach.

In this investigation, the vibration associated with a flexible connecting rod of an otherwise rigid, in-line, planar crank mechanism is considered. This problem is equivalent to determining the flexural response of a simply supported beam which is subjected to: (1) support motion which arises from the motion of the crank shaft and the mechanism kinematic constraints, (2) an axial load arising from the friction force applied to the slider end and the inertial force of the slider mass, and (3) concentrated frictional moments which exist due to the presence of bearing friction.

Similar systems have been investigated by several authors. A brief survey of past work is provided below, more complete information is found in the review articles $[5,6,7,8]$.

Neubauer et al. [9] examined the transverse deflection of an elastic connecting rod of a slider-crank mechanism by neglecting the longitudinal deformation, the Coriolis, relative tangential, and relative normal components of acceleration. By using the method of averaging and assuming a small length ratio, Jasinski et al. [10,11] investigated the dynamic stability of a flexible connecting rod in a slider-crank mechanism. Viscomi and Ayre [4] examined the nonlinear bending response of the connecting rod. Chu and Pan [1] carried out a similar study by making use of the Kantorovich method and the method of weighted residuals. Their results show that the 
effect of small viscous damping on longitudinal vibration is small and can be neglected. Moreover, a sudden increase in the amplitude of the steady state response for a small increase of the operating frequency, i.e., the jump phenomenon, was also observed. By applying a regular perturbation method to Euler-Bernoulli and Timoshenko beam models, Badlani and Kleinhenz [12] considered the dynamic stability of the undamped elastic connecting rod of an in-line slider-crank, and compared the results from each approach. Tadjbakhsh [13] introduced a general method for obtaining a single partial differential equation describing the transverse vibration of an undamped elastic link of a mechanism which contains evolutes only, using a two-parameter perturbation approach. Zhu and Chen [14] studied the stability of the response of the connecting rod by using a regular perturbation technique. Badlani and Midha [15] studied the effect of internal material damping on the dynamic response of a slider-crank mechanism by using a regular perturbation method. Both steady-state and transient solutions were determined and compared to results obtained from the undamped connecting rod. Farhang and Midha [16] have carried out a detailed study of the parameter regions in which parametric instabilities can occur in this system, and have included several modes in this study. Beale and Lee [17] have studied the motion of the flexible connecting rod of a slider-crank mechanism with a direct variational approach.

In addition of the analytical and computational works described above, some experimental work had been carried out. Golebiewski and Sadler [2] determined the bending stress at the midpoint of the elastic connecting rod analytically and experimentally for a model derived by a lumped parameter approach using d'Alembert's principle and Euler-Bernoulli beam theory. Beale et al. have recently carried out an experimental study of a system very similar to the one considered herein, and found responses consistent with their analysis [19] and the present work. Sutherland [3] studied a fully elastic, planar four-bar mechanism analytically and experimentally.

All previous stability investigations considered a nonhomogeneous Mathieu equation to describe the stability of transverse vibration, and only linear stability analyses were provided. The existence of superharmonic, subharmonic and combination resonances have been observed both in simulations and experiments $[1-4,18]$. The present investigation is aimed at providing a picture of the overall nonlinear response, with a particular focus on the superharmonic and subharmonic resonances, and their associated stability.

In the course of this study, extensive use was made of the computer assisted symbolic manipulation program Mathematica ${ }^{T M}$. This was essential in the lengthy calculations involved in the perturbation procedures. In a companion paper [20], the authors provide a detailed investigation of a discrete parameter model which is obtained from the continuous parameter model investigated here, including extensive simulation results.

This paper is organized as follows. Section 2 contains the basic assumptions and the derivation of the partial differential equations and boundary conditions used to describe the system. Section 3 provides the analysis of the equations of motion by using the method of multiple scales (MMS). Section 4 distills the results from Section 3 into a discussion of the effects of several dimensionless parameters on the system response. The paper is closed with some conclusions in Section 5 .

\section{Mathematical Modeling}

In this section, equations describing the rotary, axial and transverse dynamic responses associated with a flexible connecting rod of an otherwise rigid slider crank mechanism are derived. The basic 
assumptions which are used in deriving these equations of motion are given in Section 2.1. Based on these assumptions, the equations describing the axial and transverse deflections associated with the connecting rod, and the associated boundary conditions are derived in Section 2.2. In Section 2.3 the equations of motion and their associated boundary conditions are then converted into dimensionless form in order to minimize the number of the system parameters.

\subsection{Basic Assumptions}

In deriving the equations which describe the axial and transverse deflections associated with the flexible connecting rod, the following assumption are employed:

- Concerning the connecting rod $\mathbf{A B}$ :

1. It is an elastic member with uniform cross-section and is made of a viscoelastic material.

2. All quantities are assumed to be independent of $z$, the out-of-plane coordinate.

3. Plane cross-section remain plane after deformation.

4. It is simply supported at both ends, with rotary damping at the pivot ends (to model bearing effects).

5. Under deformation, point $\mathbf{C}$ on $\operatorname{rod} \mathbf{A B}$ moves to point $\mathbf{C}^{\prime}$; see Figure 1.

6. Shear deformation and rotary inertia are included.

- Concerning the crank element OA:

1. It is perfectly rigid.

2. It rotates with constant crank speed, $\omega$.

- Concerning the slider mass D:

1. There is no clearance and no offset.

2. There exists a velocity-dependent friction force acting between the slider and its contact surface.

3. No external forces are applied to the piston.

With these assumptions, we now proceed to derive equations which describe the axial, transverse and rotary vibrations associated with the connecting rod $\mathbf{A B}$.

\subsection{Equations of Motion}

Consider the slider-crank mechanism shown in Figure 1. The reference frames OXY and oxy represent the fixed and moving coordinate systems associated with the rigid slider-crank mechanism, respectively. Let $u(x, t)$ and $v(x, t)$ represent the axial and transverse displacements, respectively, of the connecting rod as measured in the moving coordinate system. The moving coordinate system oxy is shown in Figure 1 with its origin located at the connecting point on the crank and its $\mathbf{x}$-axis passing along the axis of the undeformed coupler. We now consider a point $\mathbf{C}$ located at $(x, 0)$ in the moving frame, which moves to point $\mathbf{C}^{\prime}$ after deformation. Modeled by the embedded geometric constraint method $[21,22]$, the position vector $\overrightarrow{\mathbf{r}}$ of Point $\mathbf{C}^{\prime}$ can be expressed as

$$
\overline{\mathbf{r}}=(x+u) \overline{\mathbf{i}}+v \overline{\mathbf{j}}
$$

where $\overline{\mathbf{i}}$ and $\overline{\mathbf{j}}$ are the unit vectors along the $\mathbf{x}$ - and $\mathbf{y}$-axes, respectively. The derivation of the 


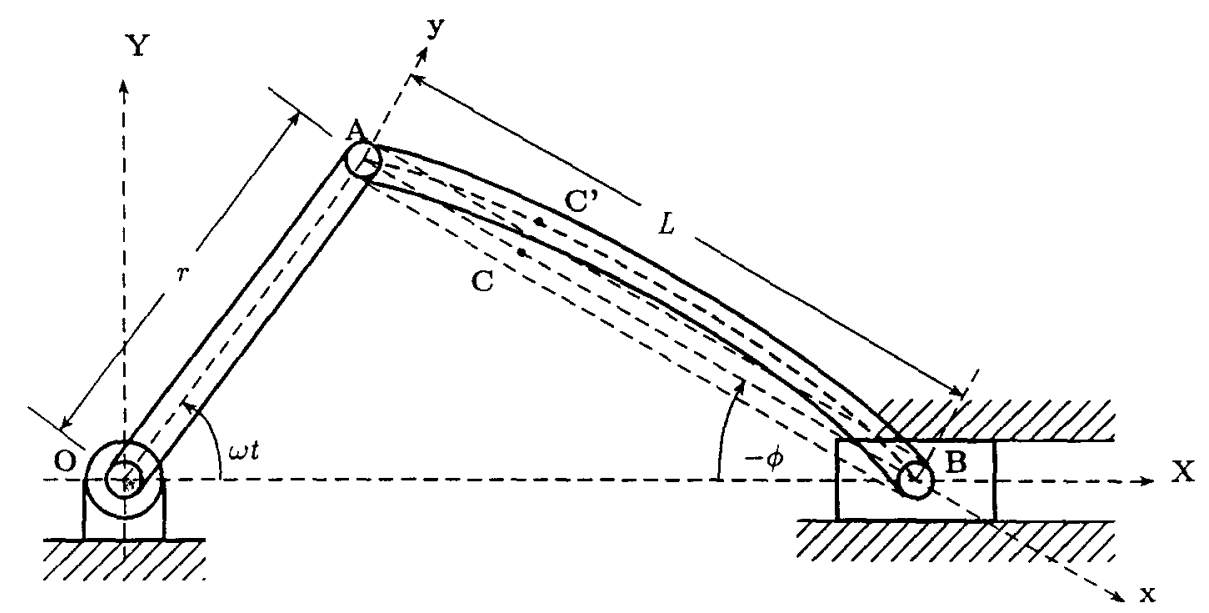

Fig. 1. Slider crank mechanism with flexible connecting rod (deformed state).

acceleration components begins with the following well-known result from dynamics [23, 24]:

$$
\overline{\mathbf{a}}=\overline{\mathbf{A}}_{a b s}+\bar{\omega} \times(\bar{\omega} \times \overline{\mathbf{r}})+\bar{\omega}_{t} \times \overline{\mathbf{r}}+\overline{\mathbf{r}}_{t t}+2 \bar{\omega} \times \overline{\mathbf{r}}_{t}=a_{x} \overline{\mathbf{i}}+a_{y} \overline{\mathbf{j}}
$$

which allows the absolute acceleration of a point to be written in terms of a moving frame of reference and where $\overline{\mathbf{a}}$ is the absolute acceleration as seen from a fixed coordinate system, $\overline{\mathbf{A}}_{a b s}$ is the absolute acceleration of the moving frame, $\overline{\mathbf{r}}$ is position vector of the particle as viewed from the moving frame, and is defined in equation (1), and $\bar{\omega}$ is the angular velocity of the moving frame.

Substituting equation (1) into equation (2), we obtain

$$
\begin{aligned}
& a_{x}=-r \omega^{2} \cos (\omega t-\phi)+u_{t t}-\phi_{t} v_{t}-\phi_{t t} v-\phi_{t}^{2}(x+u) \\
& a_{y}=-r \omega^{2} \sin (\omega t-\phi)+\phi_{t t}^{2}(x+u)+2 \phi_{t} u_{t}+v_{t t}-\phi_{t}^{2} v
\end{aligned}
$$

where a subscript $t$ represents the partial derivative with respect to the time variable, $r$ is the throw of the crank shaft, $\phi$ represents the angle between $\mathbf{A B}$ and $\mathbf{O B}$, measured positive counterclockwise, and $a_{x}$ and $a_{y}$ are the acceleration components along the $\mathbf{x}$ and $\mathbf{y}$ axes, respectively.

Consider a segment of the beam of length $\Delta x$ taken from the connecting rod as shown in Figure 2. After deformation, the angle between the median line and the $\mathbf{x}$-axis is given as

$$
\alpha=\theta+\gamma
$$

where $\theta(x, t)$ is the bending angle and $\gamma(x, t)$ is the shear angle. Let a material point located at $(x, y)$ in the undeformed state move to $\left(x^{\prime}, y^{\prime}\right)$ after deformation. Therefore

$$
\begin{aligned}
& x^{\prime}=x+u-y \sin \theta \\
& y^{\prime}=v+y \cos \theta
\end{aligned}
$$




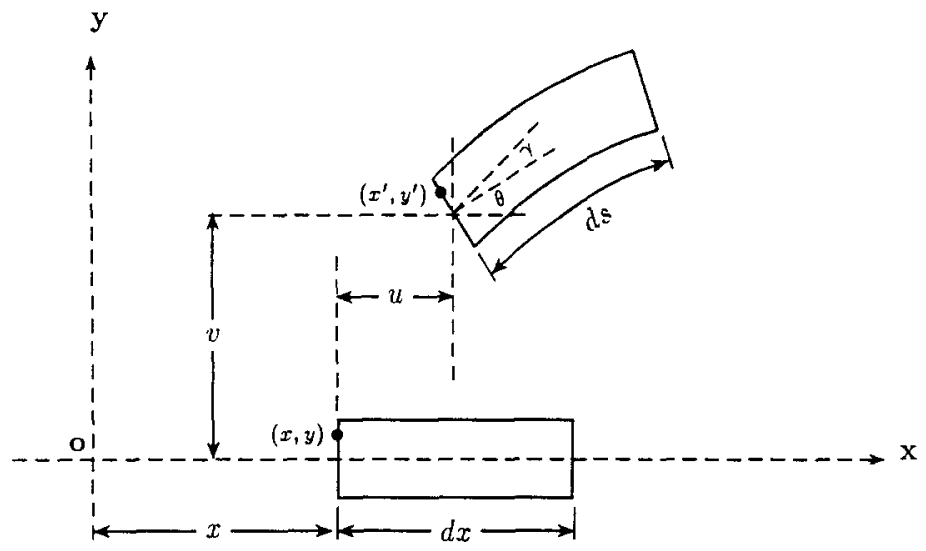

Fig. 2. Displacement of a beam element.

where $u(x, t)$ and $v(x, t)$ are the displacements of the median line along the $\mathbf{x}$ and $\mathbf{y}$ directions, respectively, and $\theta(x, t)$ is the bending angle. The length of an undeformed line element $(\mathrm{d} x, 0)$ is given in the deformed state by

$$
\mathrm{d} s=\sqrt{\mathrm{d} x^{\prime 2}+\mathrm{d} y^{\prime 2}}=\sqrt{\left[v_{x}+(y \cos \theta)_{x}\right]^{2}+\left[1+u_{x}-(y \sin \theta)_{x}\right]^{2}} \mathrm{~d} x
$$

where a subscript $x$ stands for the partial derivative with respect to the spatial variable $x$. The relative elongation in the $\mathbf{x}$-direction is defined as

$$
e=\frac{\mathrm{d} s}{\mathrm{~d} x}-1=\left(\sqrt{v_{x}^{2}+\left(1+u_{x}\right)^{2}}-1\right)-y \theta_{x}=e_{0}-y \theta_{x}
$$

where $e_{0}$ is the relative elongation of the median line. From the geometry of the deflection curve shown in Figure 2, we also have

$$
\tan \alpha=\frac{v_{x}}{1+u_{x}}, \quad \sin \alpha=\frac{v_{x}}{1+e_{0}}, \quad \cos \alpha=\frac{1+u_{x}}{1+e_{0}}
$$

where $e_{0}$ is defined in equation (9).

The connecting rod is assumed to be made of a viscoelastic material which is described by models built from discrete elastic and viscous elements. In this work, we assume that the connecting rod is made of a linear Kelvin-Voigt material. Therefore, the stress-strain-time relationship for this material is given by

$$
\sigma=E e+\mu_{c} e_{t}
$$

where $\sigma$ is the normal stress, and $e$ is the strain, $E$ is the elastic spring constant, and $\mu_{c}$ is the effective dashpot coefficient. Based on this, we obtain the following equations for the normal stress $\sigma_{x x}$ and the shear stress $\sigma_{x y}$ :

$$
\begin{aligned}
& \sigma_{x x}=E e+\mu_{c} e_{t}=E e_{0}-E y \theta_{x}+\mu_{c} e_{0, t}-\mu_{c} y \theta_{x t}, \\
& \sigma_{x y}=G \gamma .
\end{aligned}
$$


Next, we express the axial force $N$, bending moment $M$ and shear force $Q$ according to

$$
\begin{aligned}
& N=\int_{A} \sigma_{x x} \mathrm{~d} A=E A e_{0}+\mu_{c} A e_{0_{t}} \\
& M=\int_{A} \sigma_{x x} y \mathrm{~d} A=-E I \theta_{x}-\mu_{c} I \theta_{x t} \\
& Q=\int_{A} s_{x y} \mathrm{~d} A=k^{\prime} G A \gamma
\end{aligned}
$$

where $A$ is the cross-sectional area, $I$ is the area moment of the inertia of the cross-section, and $k^{\prime}$ is a shear correction factor [25].

The equations of motion are obtained by considering the balance of forces and moments in a free body diagram of the element depicted in Figure 2. From this, the following equations are derived:

$$
\begin{aligned}
& (N \cos \sigma)_{x}=(Q \sin \theta)_{x}=\rho A a_{x}, \\
& (N \sin \alpha)_{x}+(Q \cos \theta)_{x}=\rho A a_{y}, \\
& M_{x}-Q\left(1+e_{0}\right) \cos (\alpha-\theta)=-J \theta_{t t}
\end{aligned}
$$

where the acceleration components $a_{x}$ and $a_{y}$ are defined by equations (3) and (4), $\rho$ stands for the density (mass per unit volume) of the connecting rod, and $J$ is the mass moment of inertia per unit length. With equations (10) and (16), equation (19) reduces to

$$
Q=\frac{J \theta_{t t}+E I \theta_{x x}-\mu_{c} I \theta_{x x t}}{v_{x} \sin \theta+\left(1+u_{x}\right) \cos \theta}
$$

In deriving this equation, it is assumed that $I, E$, and $\mu_{c}$ are independent of $x$. From equations (5) and (16), $\alpha$ can be expressed as

$$
\alpha=\theta+\gamma=\theta+\frac{Q}{k^{\prime} A G} .
$$

The $\mathbf{x}$ and $\mathbf{y}$ components of the equation of motion are obtained by substituting equations (10), (14) and (20) into equations (17) and (18), yielding

$$
\begin{aligned}
& E A\left[\left(1+u_{x}\right)-\frac{1+u_{x}}{\sqrt{v_{x}^{2}+\left(1+u_{x}\right)^{2}}}\right]_{x}+\mu_{c} A\left[\left(1+u_{x}\right)-\frac{1+u_{x}}{\sqrt{v_{x}^{2}+\left(1+u_{x}\right)^{2}}}\right]_{x t} \\
& -\left[\frac{J \theta_{t t}-E I \theta_{x x}-\mu_{c} I \theta_{x x t}}{v_{x}+\left(1+u_{x}\right) \cot \theta}\right]_{x}=\rho A a_{x} \\
& E A\left[v_{x}-\frac{v_{x}}{\sqrt{v_{x}^{2}+\left(1+u_{x}\right)^{2}}}\right]_{x}+\mu_{c} A\left[v_{x}-\frac{v_{x}}{\sqrt{v_{x}^{2}+\left(1+u_{x}\right)^{2}}}\right]_{x t} \\
& +\left[\frac{J \theta_{t t}-E I \theta_{x x}-\mu_{c} I \theta_{x x t}}{v_{x} \tan \theta+\left(1+u_{x}\right)}\right]_{x}=\rho A a_{y} .
\end{aligned}
$$


Substituting equations (20) and (21) into equation (10), the $\theta$ equation of motion is obtained

$$
\tan \left(\theta+\frac{J \theta_{t t}-E I \theta_{x x}-\mu_{c} I \theta_{x x t}}{k^{\prime} A G\left[v_{x} \sin \theta+\left(1+u_{x}\right) \cos \theta\right]}\right)=\frac{v_{x}}{1+u_{x}} .
$$

The boundary conditions associated with these equations are derived in the following section.

\subsection{Nondimensionalization}

The equations and their associated boundary conditions are converted into dimensionless form in order to minimize the number of system parameters. To achieve this, the following dimensionless parameters are introduced:

$$
\begin{aligned}
& \bar{u}=\frac{u}{L}, \quad \bar{v}=\frac{v}{L}, \quad \bar{x}=\frac{x}{L}, \quad \xi=\frac{r}{L}, \quad S=\frac{m_{4}}{\rho A L}, \quad \alpha_{2}=\frac{E}{k^{\prime} G}, \quad \alpha_{3}=\frac{J}{\rho I}, \quad \eta^{2}=\frac{I}{A L^{2}}, \\
& \omega_{1 t}^{2}=\frac{E I \pi^{4}}{\rho A L^{4}}, \quad \Omega=\frac{\omega}{\omega_{1 t}}, \quad \bar{t}=\omega_{1 t} t, \quad \mu_{2}=\frac{\mu_{c} \omega_{1 t}}{2 E}, \quad \mu_{3}=\frac{\mu_{b} \pi^{4}}{2 \rho A L^{3} \omega_{1 t}}, \quad \mu_{4}=\frac{\mu_{s}}{2 \rho A L \omega_{1 t}} .
\end{aligned}
$$

Before proceeding to convert equations (22), (23) and (24) into dimensionless form, the physical meanings of these parameters are given. The parameter $\xi$ is the ratio of the throw length of the crank shaft to the length of the connecting rod, and is referred to hereafter as the length ratio. The parameter $S$ is the ratio of the mass of the slider to the mass of the connecting rod, and is specified as the mass ratio. The parameter $\eta=\sqrt{\frac{I}{A L^{2}}}$ represents the slenderness ratio of the connecting rod. The parameter $\alpha_{2}$ is associated with the effects of shear deformation. The parameter $\alpha_{3}$ is associated with the effects of rotary inertia. The parameter $\Omega$ is the ratio of the operating frequency to the first flexural natural frequency of the static connecting rod, $\omega_{1 t}$, and is referred to as the frequency ratio. The parameter $\mu_{2}$ represents the damping effects of the internal material damping of the connecting rod. The parameter $\mu_{3}$ represents the effects of the bearing friction applied at joints $\mathbf{A}$ and $\mathbf{B}$. The parameter $\mu_{4}$ represents the friction force acting between the slider and its contact surface.

With these dimensionless parameters, equation (22) becomes, after dropping the overbars for notational convenience,

$$
\begin{aligned}
& {\left[\left(1+u_{x}\right)-\frac{\left(1+u_{x}\right)}{\sqrt{\left(1+u_{x}\right)^{2}+v_{x}^{2}}}\right]_{x}+2 \mu_{2}\left[\left(1+u_{x}\right)-\frac{1+u_{x}}{\sqrt{\left(1+u_{x}\right)^{2}+v_{x}^{2}}}\right]_{x t}} \\
& \quad-\alpha_{3} \eta^{4} \pi^{4}\left[\frac{\theta_{t t}}{\left(1+u_{x}\right) \cot \theta+v_{x}}\right]_{x}+\eta^{2}\left[\frac{\theta_{x x}}{\left(1+u_{x}\right) \cot \theta+v_{x}}\right]_{x} \\
& \quad+2 \mu_{2} \eta^{2}\left[\frac{\theta_{x x t}}{\left(1+u_{x}\right) \cot \theta+v_{x}}\right]_{t} \\
& \quad=\eta^{2} \pi^{4}\left[-\xi \Omega^{2} \cos (\Omega t-\phi)+u_{t t}-2 \phi_{t} v_{t}-\phi_{t t} v-\phi_{t}^{2}(x+u)\right] .
\end{aligned}
$$

Under the rescaling, equation (23) becomes 


$$
\begin{aligned}
& {\left[v_{x}-\frac{v_{x}}{\sqrt{\left(1+u_{x}\right)^{2}+v_{x}^{2}}}\right]_{x}+2 \mu_{2}\left[v_{x}-\frac{v_{x}}{\sqrt{\left(1+u_{x}\right)^{2}+v_{x}^{2}}}\right]_{x t}} \\
& \quad+\eta^{4} \pi^{4} \alpha_{3}\left[\frac{\theta_{t t}}{\left(1+u_{x}\right)+v_{x} \tan \theta}\right]_{x}-\eta^{2}\left[\frac{\theta_{x x}}{\left(1+u_{x}\right)+v_{x} \tan \theta}\right]_{x} \\
& \quad-2 \mu_{2} \eta^{2}\left[\frac{\theta_{x x t}}{\left(1+u_{x}\right)+v_{x} \tan \theta}\right]_{x} \\
& \quad=\eta^{2} \pi^{4}\left[-\xi \Omega^{2} \sin \Omega t-\phi+\phi_{t t}(x+u)+2 \phi_{t} u_{t}-v_{t t}-\phi_{t}^{2} v\right],
\end{aligned}
$$

and equation (24) becomes

$$
\tan \left(\theta+\alpha_{2} \eta^{2} \frac{\alpha_{3} \eta^{2} \pi^{4} \theta_{t t}-\theta_{x x}-2 \mu_{2} \theta_{x x t}}{\left(1+u_{x}\right) \cos \theta+v_{x} \sin \theta}\right)=\frac{v_{x}}{1+u_{x}}
$$

The boundary conditions are now introduced, and these are presented in their rescaled form. The following conditions are obtained at point $\mathbf{A}$ :

$$
\begin{aligned}
& \theta(0, t)=0, \\
& v(0, t)=0, \\
& \theta_{x}(0, t)=2 \mu_{3}\left[\Omega-\phi_{t}-v_{x t}(0, t)\right] .
\end{aligned}
$$

The first and second conditions follow from the rigid crank assumption. The last condition represents the moment balance on point $\mathbf{A}$ which includes the bearing friction. The following conditions are obtained at point $\mathbf{B}$ :

$$
\begin{aligned}
& v(1, t)=-u(1, t) \tan \phi \\
& \theta_{x}(1, t)=2 \mu_{3}\left[-\phi_{t}+v_{x t}(1, t)\right] \\
& {\left[\left(1+u_{x}\right)-\frac{\left(1+u_{x}\right)}{\sqrt{\left(1+u_{x}\right)^{2}+v_{x}^{2}}}\right]+2 \mu_{2}\left[\left(1+u_{x}\right)-\frac{1+u_{x}}{\sqrt{\left(1+u_{x}\right)^{2}+v_{x}^{2}}}\right]_{t}} \\
& \quad-a_{3} \eta^{4} \pi^{4}\left[\frac{\theta_{t t}}{\left(1+u_{x}\right) \cot \theta+v_{x}}\right]+\eta^{2}\left[\frac{\theta_{x x}}{\left(1+u_{x}\right) \cot \theta+v_{x}}\right]+2 \mu_{2} \eta^{2}\left[\frac{\theta_{x x}}{\left(1+u_{x}\right) \cot \theta+v_{x}}\right]_{t} \\
& +S \eta^{2} \pi^{4}\left[-\xi \Omega^{2} \cos (\Omega t-\phi)+u_{t t}-2 \phi_{t} v_{t}-\phi_{t t} v-\phi_{t}^{2}(x+u)\right] \\
& \quad-\left[v_{x}-\frac{v_{x}}{\sqrt{\left(1+u_{x}\right)^{2}+v_{x}^{2}}}\right] \tan \phi-2 \mu_{2}\left[v_{x}-\frac{\theta_{t t}}{\sqrt{\left(1+u_{x}\right)^{2}+v_{x}^{2}}}\right]_{t} \tan \phi \\
& -\eta^{4} \pi^{4} \alpha_{3}\left[\frac{\theta_{x x}}{\left(1+u_{x}\right)+v_{x} \tan \theta}\right] \tan \phi+\eta^{2}\left[\frac{\theta_{x x}}{\left(1+u_{x}\right)+v_{x} \tan \theta}\right] \tan \phi \\
& +2 \mu_{2} \eta^{2}\left[\frac{\tan ]_{t}}{\left(1+u_{x}\right)-v_{x} \tan \theta}\right]_{t} \tan \phi \\
& -S \eta^{2} \pi^{4}\left[-\xi \Omega^{2} \sin \Omega t-\phi+\phi_{t t}(x+u)+2 \phi_{t} u_{t}-v_{t t}-\phi_{t}^{2} v\right] \tan \phi \\
& +2 \eta^{2} \pi^{4} \mu_{4} \frac{Z_{t}}{\cos \phi}+2 \eta^{2} \pi^{4} \mu_{4}\left[u_{t}-v_{t} \tan \phi\right]=0 .
\end{aligned}
$$


The first condition arises since point $\mathbf{B}$ can only move along the $\mathbf{X}$-axis. The second condition arises from the balance of the moments at point $\mathbf{B}$. Condition (33) comes from the force balance on $\mathbf{B}$ along the $\mathbf{X}$-axis.

Equations (25) and (33) form the basis of the mathematical analysis in this paper. Note that there are two main sources of nonlinearity: (1) an inertial nonlinearity arises from the inertial force associated with the slider mass and (2) a geometric nonlinearity stems from the foreshortening induced by finite transverse deformation.

\section{Analysis of the Model}

In the present section, equations (25), (26) and (27) with boundary conditions (28) to (33) are analyzed by applying the method of multiple scales [26]. To apply this method, a small parameter must be determined which will be used as a basic unit in scaling the response and the other parameters. For the current problem, the slenderness ratio $\eta$ of the flexible connecting rod is chosen to be the small parameter. The axial and transverse displacements $u(x, t)$ and $v(x, t)$ are expressed in asymptotic series in terms of $\eta$. The partial differential equations describing the dynamic behavior of the connecting rod are then expanded with these assumed solution sequences, yielding a sequence of linear partial differential equations. The dynamic response associated with the elastic connecting rod is approximated by sequentially solving these equations.

In Section 3.1, the method of multiple scales (MMS) is used to outline each individual resonance case. In Section 3.2, the case of the principal parametric resonance is considered. In Section 3.3, the case of primary resonance is examined. Superharmonic resonances of order $1 / 2$ is studied in Section 3.4 .

\subsection{Application of the Method of Multiple Scales (MMS)}

To apply MMS, we need to introduce a set of new independent time variables $T_{n}$ according to

$$
T_{n}=\epsilon^{n} t
$$

where $\epsilon$ is a small parameter which is related to the slenderness ratio of the connecting rod. The slenderness ratio of the connecting rod is scaled by defining

$$
\eta^{2}=\alpha_{1} \epsilon^{2}
$$

where $\alpha_{1}$ is a constant of $O(1)$. Moreover, the length ratio $\xi$ is scaled according to

$$
\xi=\epsilon \xi_{1}
$$

where $\xi_{1}=O(1)$. In the MMS, derivatives with respect to $t$ become expansions in terms of the partial derivatives with respect to $T_{n}$ according to

$$
\begin{aligned}
& \frac{\mathrm{d}}{\mathrm{d} t}=\frac{\partial}{\partial T_{0}}+\epsilon \frac{\partial}{\partial T_{1}}+\epsilon^{2} \frac{\partial}{\partial T_{2}}+\cdots=D_{0}+\epsilon D_{1}+\epsilon^{2} D_{2}+\cdots \\
& \frac{\mathrm{d}^{2}}{\mathrm{~d} t^{2}}=D_{0}^{2}+2 \epsilon D_{0} D_{1}+\epsilon^{2}\left(D_{1}^{2}+2 D_{0} D_{2}\right)+\cdots
\end{aligned}
$$


where $D_{j}$ stands for the partial derivative with respect to the independent time variable $T_{j}$. It is next assumed that the solutions $u(x, t)$ and $v(x, t)$ can be represented by expansions having the forms:

$$
\begin{aligned}
& u(x, t)=\epsilon u_{1}\left(x, T_{0}, T_{1}, T_{2}\right)+\epsilon^{2} u_{2}\left(x, T_{0}, T_{1}, T_{2}\right)+\epsilon^{3} u_{3}\left(x, T_{0}, T_{1}, T_{2}\right)+\cdots, \\
& v(x, t)=\epsilon v_{1}\left(x, T_{0}, T_{1}, T_{2}\right)+\epsilon^{2} v_{2}\left(x, T_{0}, T_{1}, T_{2}\right)+\epsilon^{3} v_{3}\left(x, T_{0}, T_{1}, T_{2}\right)+\cdots .
\end{aligned}
$$

Note that the number of independent time scales $T_{j}$ needed depends upon the order to which the expansions are carried out. For the present problem, it is sufficient to expand the solutions out to $O\left(\epsilon^{3}\right)$. Therefore, only the first three time scales are used in the expansions.

There are three damping parameters, $\mu_{2}, \mu_{3}$ and $\mu_{4}$, involved in equations (25), (26) and (27). These damping parameters are rescaled in such a way that they will show up in the final resonant condition together with a detuning parameter which is related to the nearness of the frequency ratio $\Omega$ to a resonance condition. A trial and error approach is used to show that the damping parameter $\mu_{2}$ must be of order $O\left(\epsilon^{2}\right), \mu_{3}$ of order $O\left(\epsilon^{2}\right)$ and $\mu_{4}$ of order $O(1)$ in order to satisfy this requirement. This leads to the following ordering of these damping coefficients in terms of $\epsilon$ :

$$
\mu_{2}=\epsilon^{2} \mu_{2_{2}}, \quad \mu_{3}=\epsilon^{3} \mu_{3_{2}}, \quad \mu_{4}=\mu_{4_{0}} .
$$

Substituting equations (35)-(41) into equation (27), expanding and gathering the like powered terms in $\epsilon$, yields the following result which relates $\theta$ to $v$ :

$$
\theta=\epsilon v_{1 x}+\epsilon^{2} v_{2 x}+\epsilon^{3}\left(v_{3 x}+\frac{v_{1 x}^{3}}{6}+\alpha_{1} \alpha_{2} v_{1 x x x}\right)+O\left(\epsilon^{4}\right)
$$

This result is used to eliminate $\theta$, yielding two dynamic equations in terms of $u$ and $v$. Substituting equations (35)-(42) into equation (9), expanding and equating the coefficients of $\epsilon^{n}$, we obtain the following expansions for the elongation $e_{0}$ of the median line along the connecting rod:

$$
\begin{aligned}
e_{0}= & \epsilon e_{0_{1}}+\epsilon^{2} e_{0_{2}}+\epsilon^{3} e_{0_{3}}+\epsilon^{4}\left(e_{0_{4}}+4 e_{0_{2}} v_{1 x}^{2}\right)+O\left(\epsilon^{5}\right) \\
= & \epsilon u_{1_{x}}+\epsilon^{2}\left(u_{2_{x}}+\frac{v_{1 x}^{2}}{2}\right)+\epsilon^{3}\left(u_{3_{x}}+v_{1_{x}} v_{2_{x}}\right) \\
& +\epsilon^{4}\left[\left(u_{4_{x}}+\frac{v_{1 x}^{4}}{8}+\frac{v_{2 x}^{2}}{2}+v_{1_{x}} v_{3_{x}}\right)-4\left(u_{2_{x}}+\frac{v_{1 x}^{2}}{3}\right) v_{1 x}^{2}\right]+O\left(\epsilon^{5}\right)
\end{aligned}
$$

where $e_{0_{j}}$ represents the $j$-th order term in the expansion of $e_{0}$ which is defined in equation (9). Applying the same procedure to the equations of motion (25)-(27), the following sequence of equations is obtained:

\section{Order $\epsilon$}

$$
u_{1_{x x}}=\left(e_{0_{1}}\right)_{x}=0
$$


Order $\epsilon^{2}$

$$
\left(u_{2_{x}}+\frac{v_{1 x}^{2}}{2}\right)_{x}=\left(e_{0_{2}}\right)_{x}=0,
$$

Order $\epsilon^{3}$

$$
\begin{aligned}
& \left(u_{3_{x}}+v_{1_{x}} v_{2_{x}}\right)_{x}=\left(e_{0_{3}}\right)_{x}=-\alpha_{1} \pi^{4} \xi_{1} \Omega^{2} \cos \Omega T_{0}, \\
& \alpha_{1} \pi^{4} D_{0}^{2} v_{1}+\alpha_{1} v_{1_{x x x x}}=\left(e_{0_{2}} v_{1_{x}}\right)_{x}+\alpha_{1} \pi^{4} \xi_{1} \Omega^{2}(1-x) \sin \Omega T_{0},
\end{aligned}
$$

\section{Order $\epsilon^{4}$}

$$
\begin{gathered}
\left(e_{0_{4}}\right)_{x}+2 \mu_{2_{2}}\left(D_{0} e_{0_{2}}\right)_{x}-\left(e_{0_{2}} v_{1 x}^{2}\right)_{x}+\alpha_{1}\left(v_{1 x} v_{1 x x x}\right)_{x}=\alpha_{1} \pi^{4}\left[D_{0}^{2} u_{2}-\xi_{1} \Omega^{2} v_{1} \sin \Omega T_{0}\right. \\
\left.-\xi_{1} \Omega^{2} v_{1} \sin \Omega T_{0}+2 \xi_{1} \Omega\left(D_{0} v_{1}\right) \cos \Omega T_{0}+\xi_{1}^{2} \Omega^{2} \sin ^{2} \Omega T_{0}-\xi_{1}^{2} \Omega^{2} x \cos ^{2} \Omega T_{0}\right], \\
\alpha_{1} \pi^{4} D_{0}^{2} v_{2}+\alpha_{1} v_{2 x x x x}= \\
=\left(e_{0} v_{1 x}\right)_{x}+\left(e_{0_{2}} v_{2 x}\right)_{x}-2 \alpha_{1} \pi^{4}\left(D_{0} D_{1} v_{1}\right) \\
+\alpha_{1} \pi^{4} \xi_{1}^{2} \Omega^{2} \cos \Omega T_{0} \sin \Omega T_{0},
\end{gathered}
$$

By applying the same procedure to the boundary conditions, the following sequence of boundary condition equation is determined:

\section{Order $\epsilon$}

$$
\begin{aligned}
& u_{1}=v_{1}=v_{1_{x x}}=0, \quad \text { at } x=0, \\
& u_{1 x}=v_{1}=v_{1 x x}=0, \quad \text { at } x=1,
\end{aligned}
$$

\section{Order $\epsilon^{2}$}

$$
\begin{aligned}
& u_{2}=v_{2}=v_{2 x x}=0, \quad \text { at } x=0, \\
& \left(u_{2 x}+\frac{v_{1 x}^{2}}{2}\right)=v_{2}=0, \quad v_{2 x x}=-2 \mu_{3_{2}} \Omega, \quad \text { at } x=1,
\end{aligned}
$$

Order $\epsilon^{3}$

$$
\begin{aligned}
& u_{3}=v_{3}=0, \\
& v_{3 x x}=-\alpha_{1} \alpha_{2} v_{1 x x x x x}-v_{1 x}^{2} \frac{v_{1 x x x}}{2}-2 \mu_{3} D_{0} v_{1 x}-2 \mu_{3} \xi_{1} \Omega \cos \Omega T_{0}, \quad \text { at } x=0, \\
& v_{3}=0, \\
& \left(u_{3 x}+v_{1 . x} v_{2 x}\right)=S \alpha_{1} \pi^{4} \xi_{1} \Omega^{2} \cos \Omega T_{0}+2 \alpha_{1} \pi^{4} \mu_{4_{0}} \xi_{1} \Omega \sin \Omega T_{0}, \\
& v_{3 x x}=-\alpha_{1} \alpha_{2} v_{1 . x x x x}-v_{1 . x}^{2} \frac{v_{1 x x}}{2}-2 \mu_{3} D_{0} v_{1 x}+2 \mu_{3} \xi_{1} \Omega \cos \Omega T_{0}, \quad \text { at } x=1 .
\end{aligned}
$$


It can be shown that $u_{1}\left(x, T_{0}, T_{1}, T_{2}\right)=0$, and hence all the terms involving products with $u_{1}\left(x, T_{0}, T_{1}, T_{2}\right)$ are dropped. This result is obtained by first solving equation (44) which gives $u_{1}\left(x, T_{0}, T_{1}, T_{2}\right)=b_{1}\left(T_{0}, T_{1}, T_{2}\right) x=b_{2}\left(T_{0}, T_{1}, T_{2}\right)$. By applying boundary conditions (50) and (51), it is seen that $b_{1}=b_{2}=0$. Hence, $u_{1}\left(x, T_{0}, T_{1}, T_{2}\right)$ must be zero. This indicates that, for a simply-supported, slender beam, the axial deformation is much smaller than the transverse deformation.

Next, we consider equation (45) and its associated boundary condition (53). Together, they reveal that

$$
\left(u_{2 x}+\frac{v_{1 x}^{2}}{2}\right)=e_{0_{2}}=0 .
$$

Consequently, equation (47) reduces to the following partial differential equation for $v_{1}$ :

$$
\alpha_{1} \pi^{4}\left(D_{0}^{2} v_{1}\right)+\alpha_{1} v_{1 x x x x}=\alpha_{1} \pi^{4} \xi_{1} \Omega^{2}(1-x) \sin \Omega T_{0}
$$

which can be converted into the following ordinary differential equation:

$$
\left(D_{0}^{2} \hat{v}_{1 n}\right)+\omega_{n}^{2} \hat{v}_{1 n}=\frac{2}{n \pi} \xi_{1} \Omega^{2} \sin \Omega T_{0}
$$

by Galerkin's procedure with $v_{1}=\hat{v}_{1 n}(\sin n \pi x)$, where $\omega_{n}^{2}=n^{4}$ for $n=1,2,3, \ldots$ (In this work, results for $n=1$ are emphasized, since these are the most important in practice [4].) In analyzing the particular solution of $\hat{v}_{1 n}$, we need to consider the following cases separately: (1) $\Omega$ is near $\omega_{n}$ and (2) $\Omega$ is away from $\omega_{n}$. The case in which $\Omega$ is near $\omega_{n}$ corresponds to the primary resonance and is investigated in Section 3.3. For the present, it is assumed that $\Omega$ is away from $\omega_{n}$ and the analysis is carried to higher orders. When $\Omega$ is away from $\omega_{n}$, the general solution of $\hat{v}_{1 n}$ is given as

$$
\hat{v}_{1 n}=A_{n}\left(T_{1}, T_{2}\right) \exp \left(j \omega_{n} T_{0}\right)+\Lambda_{1} \sin \Omega T_{0}+c . c .
$$

where c.c. stands for the complex conjugate of the preceding term, and

$$
\Lambda_{1}=\left(\frac{2}{n \pi}\right) \frac{\xi_{1} \Omega^{2}}{\left(\omega_{n}^{2}-\Omega^{2}\right)}
$$

represents the amplitude of the particular solution. Equation (46), together with its boundary condition (55), leads to

$$
e_{0_{3}}=\left(u_{3 x}+v_{1 x} v_{2 x}\right)=\alpha_{1} \pi^{4}(1-x+S) \xi_{1} \Omega^{2} \cos \Omega T_{0}+2 \alpha_{1} \pi^{4} \xi_{1} \Omega \mu_{4_{0}} \sin \Omega T_{0}
$$

which represents the third order expansion of the axial elongation of the median line along the connecting rod. Substituting equations (56) and (61) into equation (49), the following partial differential equation for $v_{2}$ is obtained:

$$
\begin{aligned}
& \alpha_{1} \pi^{4} D_{0}^{2} v_{2}+\alpha_{1} v_{2 x x x x}=\alpha_{1} \pi^{4} \xi_{1} \Omega^{2}\left[(1-x+S) v_{1 x}\right]_{x} \cos \Omega T_{0}-2 \alpha_{1} \pi^{4} D_{0} D_{1} v_{1} \\
& \quad+\alpha_{1} \pi^{4} \xi_{1}^{2} \Omega^{2} \cos \Omega T_{0} \sin \Omega T_{0}+2 \alpha_{1} \pi^{4} \mu_{4_{0}} \xi_{1} \Omega v_{1 x x} \sin \Omega T_{0}
\end{aligned}
$$


which can be converted into the following ordinary differential equation:

$$
\begin{aligned}
& D_{0}^{2} \hat{v}_{2 n}+\omega_{n}^{2} \hat{v}_{2 n}=-2 j \omega_{n}\left(D_{1} A_{n}\right) \exp \left(j \omega_{n} T_{0}\right)+\left(\frac{1-\cos n \pi}{n \pi}\right) \xi_{1}^{2} \Omega^{2} \sin \left(2 \Omega T_{0}\right) \\
& \quad-\frac{1}{4}(1+2 S)(n \pi)^{2} \xi_{1} \Omega^{2}\left[A_{n} \exp \left(j \omega_{n} T_{0}+j \Omega T_{0}\right)+\bar{A}_{n} \exp \left(-j \omega_{n} T_{0}+j \Omega T_{0}\right)\right] \\
& \quad-\mu_{4_{4}} \xi_{1} \Omega\left[(n \pi)^{2} \bar{A}_{n} \exp \left(j \Omega T_{0}-j \omega T_{0}-j \pi / 2\right)-\frac{(n \pi)^{2}}{2} \Lambda_{1} \exp \left(2 j \Omega T_{0}\right)\right] \\
& \quad-\frac{1}{4}(1+2 S)(n \pi)^{2} \Lambda_{1} \xi_{1} \Omega^{2} \sin \left(2 \Omega T_{0}\right)
\end{aligned}
$$

using $v_{2}=\hat{v}_{2 n}(\sin n \pi x)$, where an overbar stands for the complex conjugate of the corresponding term. Now, in analyzing the solution of this equation, we need to distinguish between the following cases: (1) $2 \Omega$ is near $\omega_{n}$, (2) $\Omega$ is near $2 \omega_{n}$ and (3) $\Omega$ is away from $2 \omega_{n}$ and $\frac{\omega_{n}}{2}$. The first case corresponds a superharmonic resonance, and is investigated in Section 3.4. The second case corresponds the principal parametric resonance which is investigated in Section 3.2. At the present time, it is assumed that $\Omega$ is away from $2 \omega_{n}$ and $\frac{\omega_{n}}{2}$. Under this condition, we must have $D_{1} A_{n}=0$ in order to remove the secular term from the particular solution of $\hat{v}_{2 n}$. This implies that the amplitude $A_{n}$ of the free oscillation term in the general solution of $v_{1}$ (equation (59)) must be independent of the time scale $T_{1}=\epsilon t$. The particular solution of $\hat{v}_{2 n}$ is then given by

$$
\begin{aligned}
\hat{v}_{n}= & \Lambda_{21} \sin 2 \Omega T_{0}+\Lambda_{22} \exp \left(j \Omega T_{0}+j \omega_{n} T_{0}\right)+\Lambda_{23} \exp \left(j \Omega T_{0}-j \omega_{n} T_{0}\right) \\
& +\Lambda_{24} \exp \left(2 j \Omega T_{0}\right)+\Lambda_{25} \exp \left(j \Omega T_{0}-j \omega_{n} T_{0}-j \pi / 2\right)+c . c .
\end{aligned}
$$

where

$$
\begin{aligned}
& \Lambda_{21}=\frac{1-\cos n \pi}{n \pi} \frac{\xi_{1}^{2} \Omega^{2}}{\left(\omega_{n}^{2}-4 \Omega^{2}\right)}-\frac{1}{4}(1+2 S)(n \pi)^{2} \Lambda_{1} \frac{\xi_{1} \Omega^{2}}{\omega_{n}^{2}-4 \Omega^{2}}, \\
& \Lambda_{22}=-\frac{(1+2 S)}{4}(n \pi)^{2} \xi_{1} \Omega^{2} \frac{A_{n}}{\left[\omega_{n}^{2}-\left(\omega_{n}+\Omega\right)^{2}\right]}, \\
& \Lambda_{23}=-\frac{1}{4}(1+2 S)(n \pi)^{2} \xi_{1} \Psi^{2} \frac{\bar{A}_{n}}{\left[\omega_{n}^{2}-\left(\omega_{n}-\Omega\right)^{2}\right]}, \\
& \Lambda_{24}=\frac{\Lambda_{1}}{2} \frac{(n \pi)^{2}}{\omega_{n}^{2}-4 \Omega^{2}},
\end{aligned}
$$

and

$$
\Lambda_{25}=-\mu_{4_{0}} \xi_{1} \Omega(n \pi) \frac{\bar{A}_{n}}{\omega_{n}^{2}-\left(\Omega-\omega_{n}\right)^{2}} .
$$

From the expression $u_{2 x}+\frac{v_{1 x}^{2}}{2}=0, u_{2}\left(x, T_{0}, T_{1}, T_{2}\right)$ is solved for, yielding

$$
u_{2}=-\frac{1}{4}(n \pi)^{2} \hat{v}_{1 n}^{2}\left(x+\frac{\sin 2 n \pi x}{2 n \pi}\right)
$$

where $\hat{v}_{1 n}$ is given by equation (59). 
At this point, we pause and discuss some general features of this pattern of analysis. After a small, constant parameter $\epsilon$ has been introduced, and solutions are expressed in uniform expansions based on this parameter. By using these assumed solution sequences, the equations describing the transverse and the axial deflection can be converted into a sequence of partial differential equations which can be solved easily, since they are linear due to the nature of the perturbation method. In solving these equations, the primary resonance arises first and then the principal parametric resonance. After these two resonances, several secondary resonances arise. In principle, it is possible to extend this analysis to study higher order resonances, for instance the superharmonic resonance which occurs when $\Omega$ is near $\omega_{n} / 3$. Hsieh [28] had extended the analysis pattern to study the superharmonic and subharmonic resonances of order 3 . For the sake of brevity, only the first few resonances are considered here. In all cases, this approach involves a significant amount of symbolic computation. The present results were accomplished with a great deal of help from the computer-assisted symbolic manipulation program Mathematica ${ }^{T M}$.

Although the primary resonance occurs first in the analysis, the principle parametric resonance is investigated first, since this pattern will simplify the analysis and make it more easily understood. The superharmonic resonance is presented after these two cases.

\subsection{Principal Parametric Resonance}

When the frequency ratio $\Omega$ is near $2 \omega_{n}$, a principal parametric resonance takes place. To describe the nearness of $\Omega$ to $2 \omega_{n}, \Omega$ is expressed as $\Omega=2 \omega_{n}+2 \epsilon \sigma_{1}$ where $\sigma_{1}$ is the detuning parameter. At the same time, the damping parameter $\mu_{2}$ is rescaled as $\mu_{2}=\epsilon \mu_{2}$. We first expand equations (25), (26) and (27) with these ordering relations, and then carry out the procedure described in the previous section. Since the computational work is routine, only a few essential results are presented in the following. The detailed solution procedure can be found in [28].

Following the same procedure described in the previous section, the first order flexural vibration associated with the connecting rod is described by equation (58). Consequently, the general solution of $\hat{v}_{1 n}$ is given by equation (59). When solving the second order equation (63) for $\tilde{v}_{2 n}$, the following equation is obtained in removing the secular terms from the particular solution of $\hat{v}_{2 n}$.

$$
\begin{aligned}
& -2 j \omega_{n}\left(D_{1} A_{n}\right)-2 j \mu_{2_{1}} \omega_{n} A_{n} n^{4}-(1-2 S)(n \pi)^{2} \omega_{n}^{2} \bar{A}_{n} \xi_{1} \exp \left(2 j \sigma_{1} T_{1}\right) \\
& -2 \mu_{4_{0}}(n \pi)^{2} \omega_{n} \bar{A}_{n} \xi_{1} \exp \left(2 j \sigma_{1} T_{1}-j \frac{\pi}{2}\right)=0 .
\end{aligned}
$$

Expanding equation (71) with $A_{n}\left(T_{1} T_{2}\right)=\frac{a}{2} \exp (j \Psi)$, and separating the resulting equation into real and imaginary parts, we obtain

$$
\begin{aligned}
& a^{\prime}=-\mu_{2_{1}} n^{4} a-2 a \xi_{1} \Delta_{1} \sin 2 \Phi_{1}+a(n \pi)^{2} \xi_{1} \mu_{4_{0}} \cos 2 \Phi_{1} \\
& a \Phi_{1}^{\prime}=\sigma_{1} a-2 a \xi_{1} \Delta_{1} \cos 2 \Phi_{1}-a(n \pi)^{2} \xi_{1} \mu_{4_{0}} \sin 2 \Phi_{1}
\end{aligned}
$$

where the phase angle $\Phi_{1}$ and parameter $\Delta_{1}$ are defined by

$$
\begin{aligned}
& \Phi_{1}=\sigma_{1} T_{1}-\Psi, \\
& \Delta_{1}=\frac{(1+2 S)}{4}(n \pi)^{2} n^{2}
\end{aligned}
$$


and the primes indicate derivatives with respect to the time scale $T_{1}$. Periodic steady state conditions are achieved whenever $a^{\prime}=a \Phi_{1}^{\prime}=0$. These yield the frequency response equation which takes the form:

$$
\left[\sigma_{1}^{2}+n^{8} \mu_{21}^{2}-\left(2 \xi_{1} \Delta_{1}\right)^{2}-(n \pi)^{4} \mu_{4_{11}}^{2} \xi_{1}^{2}\right] a^{2}=0 .
$$

From this frequency response equation, it is noted that the trivial solution $a=0$ is always a steady state response for this resonance. To determine the stability associated with this trivial solution, equation (71) is transformed into Cartesian coordinates by substituting $A_{n}=\left(B_{R}+\right.$ $\left.j B_{1}\right) \exp \left(j \sigma_{1} T_{1}+\gamma T_{1}\right)$, and the resulting equation is separated into real and imaginary parts. From the resulting two equations, the nontrivial solutions of $B_{R}$ and $B_{l}$ are determined. From this, it is found that when

$$
\sigma_{1}^{2}+n^{8} \mu_{z_{1}}^{2}>\xi_{1}^{2}\left[4 \Delta_{1}^{2}-\left(n^{2} \pi^{2} \mu_{t_{1}}\right)^{2}\right]
$$

the trivial solution is unstable. Otherwise, it is stable. Figure 3 shows an example of a stability boundary in the $\xi-\Omega$ plane for this resonance.

In order to capture the effects of the nonlinearities, we need to rescale either the solutions or the parameters. so that the nonlinearity will be included in the final equation describing the resonance condition. In order to achieve this, it is useful to note that this problem is equivalent to a simply supported beam subjected to both transverse and axial forces which arise from the same source. This source is the non-inertial frame in which the connecting rod moves, and these inertial forces are proportional to the length ratio $\xi$. Therefore, the length ratio is rescaled in order to extend the analysis to include the nonlinearity.

The analysis is restarted by letting $\xi=\epsilon^{2} \xi_{2}$ while keeping the damping parameters as given in equation (41). Expanding equations (25)-(27) and their associated boundary conditions with this length ratio, and equating the coefficients of like-powered terms in $\epsilon$, a new sequence of linear partial differential equations is obtained. Following the usual procedure, the equation describing the first order flexural vibration becomes

$$
D_{0}^{2} \hat{v}_{1 n}+\omega_{n}^{2} \hat{v}_{1 n}=0
$$

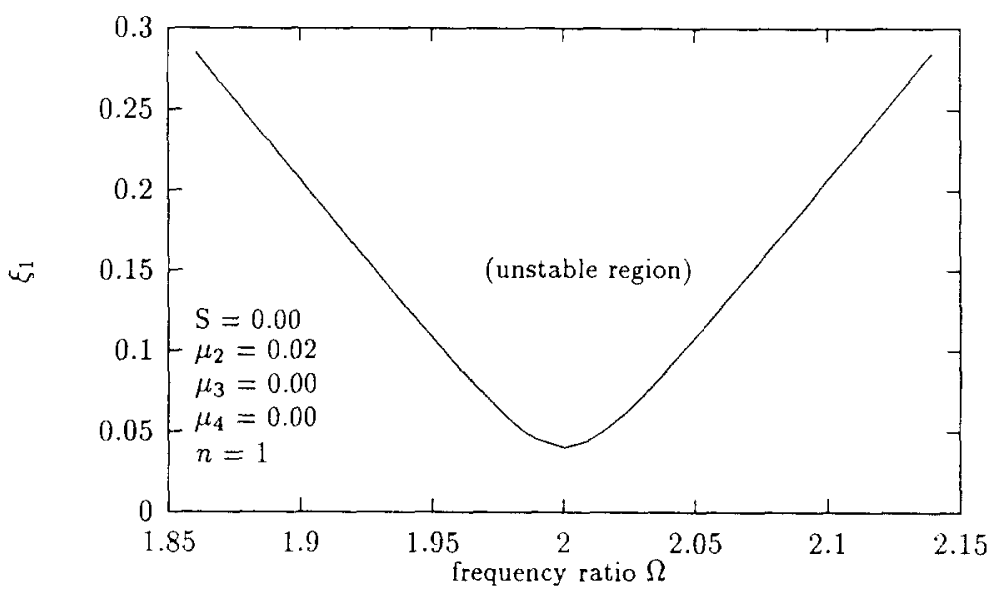

Fig. 3. Stability boundary for the principal parametric resonance. 
which admits the following solution

$$
\hat{v}_{1 n}=A_{n} \exp \left(j \omega_{n} T_{0}\right)+c . c .
$$

From this, the following ordinary differential equation describing the second order flexural vibration is obtained:

$$
D_{0}^{2} \hat{v}_{2 n}+\omega_{n}^{2} \hat{v}_{2 n}=\frac{2}{n \pi} \xi_{2} \Omega^{2} \sin \Omega T_{0}-2 j \omega_{n}\left(D_{1} A_{n}\right) \exp \left(j \omega_{n} T_{0}\right)+c . c .
$$

In analyzing the particular solution of $\hat{v}_{2 n}$, we need to distinguish the following two cases: (1) $\Omega$ is near $\omega_{n}$ and (2) $\Omega$ is away from $\omega_{n}$. The first case is referred to as the primary resonance, which is investigated in Section 3.3. For the present, the analysis is continued to third order with the assumption that $\Omega$ is away from $\omega_{n}$. Under this condition, $D_{1} A_{n}=0$ must hold in order to remove the secular term from the particular solution of equation (80). This implies that the amplitude $A_{n}$ must be independent of the time scale $T_{1}$. In removing the secular terms from the equation describing the third order flexural vibration, the following resonance condition is obtained:

$$
\begin{aligned}
& -2 j \omega_{n}\left(D_{2} A_{n}\right)-2 j \omega_{n} n^{4} \mu_{2_{2}} A_{n}+2 j \frac{n^{4}}{\pi^{2}} A_{n} \mu_{3_{2}}-j(n \pi)^{4} \mu_{4_{0}} A_{n}^{2} \bar{A}_{n} \omega_{n} \\
& +\xi_{2}(n \pi)^{2} \mu_{4_{0}} \Omega \bar{A}_{n} \exp \left(2 j \sigma_{2} T_{1}-j \pi / 2\right)+A_{n}(n \pi)^{2} n^{4}\left[\alpha_{1} \alpha_{2}+\alpha_{1} \alpha_{3}\right] \\
& -\frac{1}{4}(1+2 S)(n \pi)^{2} \xi_{2} \Omega^{2} \bar{A}_{n} \exp \left(2 j \sigma_{2} T_{1}\right)-\left[\frac{15}{8}-\left(\frac{1}{3}+S\right)(n \pi)^{2}\right](n \pi)^{2} n^{4} A_{n}^{2} \bar{A}_{n}=0
\end{aligned}
$$

where $\Omega=2+2 \epsilon^{2} \sigma_{2}$. Expanding this equation with $A_{n}=\frac{a}{2} \exp (j \Psi)$, and separating the resulting equation into real and imaginary parts, yields

$$
\begin{aligned}
& a^{\prime}=-n^{4} \mu_{2_{2}} a-\frac{1}{8}(n \pi)^{4} \mu_{4_{0}} n^{2} a^{3}+\frac{n^{2}}{\pi^{2}} \mu_{3_{2}} a-2 a \xi_{2} \Delta_{1} \sin 2 \Phi_{2}+a \mu_{4_{0}} \xi_{2}(n \pi)^{2} \cos 2 \Phi_{2}, \\
& a \Phi_{2}^{\prime}=\sigma_{2} a+\Delta_{3} a-\Delta_{2} a^{3}-2 a \Delta_{1} \xi_{2} \cos 2 \Phi_{2}-a \mu_{4_{0}} \xi_{2}(n \pi)^{2} \sin 2 \Phi_{2}
\end{aligned}
$$

where

$$
\begin{aligned}
& \Delta_{2}=\frac{1}{8}(n \pi)^{2} n^{2}\left[\frac{15}{8}-\left(\frac{1}{3}+S\right)(n \pi)^{2}\right], \\
& \Delta_{3}=\frac{n^{2}}{2}(n \pi)^{2}\left(\alpha_{1} \alpha_{2}+\alpha_{1} \alpha_{3}\right), \\
& \Phi_{2}=\sigma_{2} T_{2}-\Psi
\end{aligned}
$$

and primes indicate derivatives with respect to the time scale $T_{2}$. The steady state condition can be obtained by letting $a^{\prime}=a \Phi_{2}^{\prime}=0$, which leads to the following frequency response equation:

$$
\begin{aligned}
& a^{2}\left[\left(n^{4} \mu_{2_{2}}+\frac{1}{8}(n \pi)^{4} \mu_{4_{0}} \omega_{n} a^{2}-\frac{n^{2}}{\pi^{2}} \mu_{3_{2}}\right)^{2}+\left(\sigma_{2}-\Delta_{2} a^{2}+\Delta_{3}\right)^{2}\right. \\
& \left.-\left(2 \xi_{2} \Delta_{1}\right)^{2}-(n \pi)^{4} \xi_{2}^{2} \mu_{4_{0}}^{2}\right]=0 .
\end{aligned}
$$


In addition to the trivial response, $a=a_{1}=0$, it is now possible to solve for the nontrivial solutions of $a^{2}$. These are given by:

$$
\begin{aligned}
& \left(a_{2}\right)^{2}=\left(\frac{1}{k}\right)\left[-l+\sqrt{l^{2}-k m}\right], \\
& \left(a_{3}\right)^{2}=\left(\frac{1}{k}\right)\left[-l-\sqrt{l^{2}-k m}\right],
\end{aligned}
$$

where

$$
\begin{aligned}
& k=\left[\left(\frac{\mu_{4_{0}}}{8}\right)(n \pi)^{4}\right]^{2}+\Delta_{2}^{2} \\
& l=\left(\frac{\mu_{4_{0}}}{8}\right)(n \pi)^{4}\left[n^{4} \mu_{2_{2}}-\left(\frac{n}{\pi}\right)^{2} \mu_{3_{2}}\right]-\left(\sigma_{2}-\Delta_{3}\right) \Delta_{2}, \\
& m=\left[n^{4} \mu_{2_{2}}-\left(\frac{n}{\pi}\right)^{2} \mu_{3_{2}}\right]^{2}+\left(\sigma_{2}-\Delta_{3}\right)^{2}-4 \xi_{2}^{2} \Delta_{1}^{2}-(n \pi)^{4} \xi_{2}^{2} \mu_{4_{0}}^{2}
\end{aligned}
$$

In order for amplitudes $a_{2}$ and $a_{3}$ to be real, the term inside the radical must be positive or zero. This implies that, in order to produce a sustained nontrivial steady state response, the magnitude of the forcing must be large enough to overcome the effects of dissipation. Specifically, the following must hold

$$
\sigma_{2}+\Delta_{3}<\Gamma=\sqrt{\left(2 \xi_{2} \Delta_{1}\right)^{2}+(n \pi)^{4} \xi_{2}^{2} \mu_{4_{0}}^{2}+\left[\mu_{22} n^{4}-\left(\frac{n}{\pi}\right)^{2} \mu_{3_{2}}\right]^{2}}
$$

for the existence of $\left(a_{2}\right)^{2}$, and

$$
\sigma_{2}+\Delta_{3}<-\Gamma=-\sqrt{\left(2 \xi_{2} \Delta_{1}\right)^{2}+(n \pi)^{4} \xi_{2}^{2} \mu_{4_{0}}^{2}-\left(\mu_{22} n^{4}-\left(\frac{n}{\pi}\right)^{2} \mu_{3_{2}}\right)^{2}}
$$

must hold for the existence of $\left(a_{3}\right)^{2}$. From the eigenvalues of the Jacobian matrix associated with equations (82) and (83), we obtain the following conclusions:

(1) when $\sigma_{2}>-\Delta_{3}+\Gamma$ there is only one response, the trivial one, and it is stable,

(2) when $-\Delta_{3}-\Gamma<\sigma_{2}<-\Delta_{3}+\Gamma$. the trivial solution becomes unstable, while $a_{2}$ exists and is stable,

(3) when $\sigma_{2}<-\Delta_{3}-\Gamma a_{3}$ exists and is unstable, while the trivial solution and $a_{2}$ exist and are stable.

An example frequency response of $a$ vs. $\Omega$ determined from equation (87) is plotted in Figure 4 .

REMARKS. Before leaving this section, a few points should be made. First, we compare the linear and nonlinear results. If one neglects the effects of shear deformation $\alpha_{2}$, rotary inertia $\alpha_{3}$, the friction force introduced by elastic deformation (i.e., the $\mu_{4} \alpha^{3}$ term) and the bearing damping $\mu_{3}$, then equations (82) and (83) become

$$
\begin{aligned}
& a^{\prime}=-n^{4} \mu_{2_{2}} a-2 a \xi_{2} \Delta_{1} \sin \left(2 \Phi_{2}\right)+a \mu_{4_{0}} \xi_{2}(n \pi)^{2} \cos \left(2 \Phi_{2}\right), \\
& a \Phi_{2}^{\prime}=\sigma_{2} a-\Delta_{2} a^{3}-2 a \Delta_{1} \xi_{2} \cos \left(2 \Phi_{2}\right)-a \mu_{4_{n}} \xi_{2}(n \pi)^{2} \sin \left(2 \Phi_{2}\right) .
\end{aligned}
$$




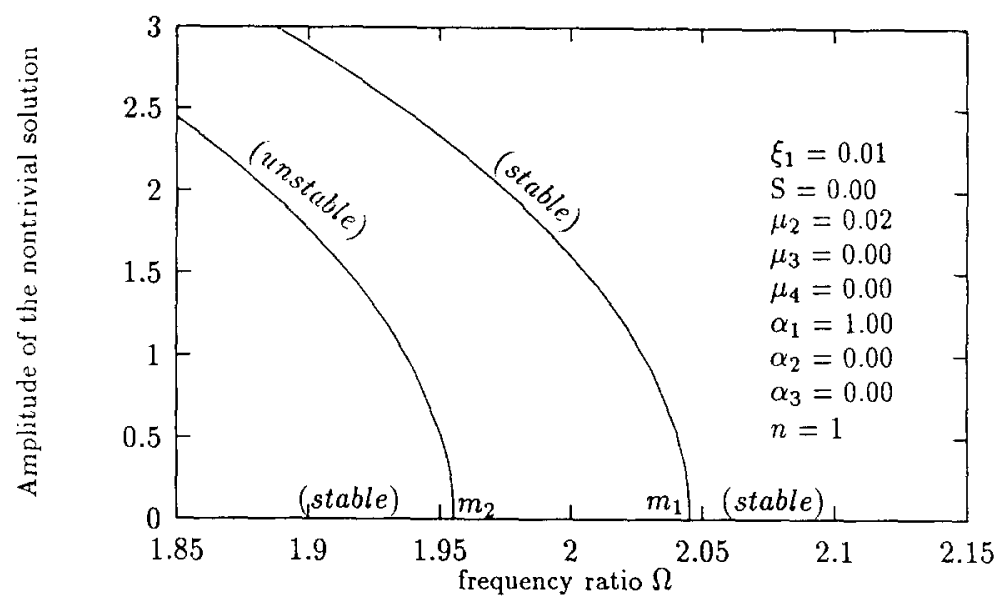

Fig. 4. Frequency response equation for the principal parametric resonance.

In consequence, the frequency response equation becomes

$$
a^{2}\left[\left(n^{4} \mu_{2_{2}}\right)^{2}+\left(\sigma_{2}-\Delta_{2} a^{2}\right)^{2}-\left(2 \xi_{2} \Delta_{1}\right)^{2}-(n \pi)^{4} \xi_{2}^{2} \mu_{4_{0}}^{2}\right]=0 \text {. }
$$

It is clear that equations (76) and (97) are identical in their linear parts. To show this, substitute $\mu_{2_{2}}=\epsilon \mu_{2_{1}}$ and $\xi_{2}=\epsilon \xi_{1}$, into equation (97). Then equation (97) reduces to equation (76) as $a$ approaches zero. Moreover, the equation describing the variation of the amplitude of the response (equations (72) and (96)) are of the same form. The difference between the linear and nonlinear analyses appears in the equation describing the variation of the phase angle $\Phi_{2}$ (equations (73) and (96)). This implies that the primary effect of the nonlinearity is to affect the phase angle. From another point of view, the nonlinearity affects the rate at which energy is driven into the system. In the absence of nonlinearity, the response is unstable when the parameters are located inside the unstable regions, and according to the linear theory, the amplitude will grow without limit. However, this increasing amplitude will be accompanied with a change of phase angle due to the presence of the nonlinearities. A new steady state condition will then be achieved when the energy put into the system is balanced by the energy dissipated by the damping. This is typical of results obtained from nonlinear Mathieu equations [26].

Furthermore, consider the main nose of instability, that is, the region in which the trivial solution becomes unstable. If we substitute the trivial solution $a=0$ into equation (97), equation (76) is recovered. Therefore, as expected, the same region of instability of the trivial solution is obtained from the linear and nonlinear analyses.

Finally, consider effects of shear deformation and rotary inertia, which are included in the parameter $\Delta_{3}$. The shear deformation and rotary inertia terms, although linear, do not show up to the first order solution. However, they do contribute in the higher order analysis and affect the response by shifting the instability region along the frequency axis to the left by a constant amount which is independent of the amplitude of the response (see the $\left(\Delta_{3}+\sigma_{2}\right)$ term in equation (83)).

\subsection{Primary Resonance $\left(\Omega \approx \omega_{n}\right)$}

The case in which the frequency $\Omega$ is near $\omega_{n}$, i.e., the primary resonance, is now examined. To 
begin the analysis, reconsider equation (80) in Section 3.2, and assume that $\Omega$ is near $\omega_{n}$. Moreover, the damping parameter $\mu_{2}$ is rescaled as $\mu_{2}=\epsilon \mu_{2}$ and $\mu_{4}$ is rescaled as $\mu_{4}=\mu_{4_{1},}$. Under these conditions, in order to remove the secular term from the particular solution of $\dot{v}_{2 n}$, the following must hold:

$$
-2 j \omega_{n}\left(D_{1} A_{n}\right)-\frac{\xi_{2}}{n \pi} \Omega^{2} \exp \left(j \sigma_{1} T_{1}-j \frac{\pi}{2}\right)-2 j \mu_{21} n^{4} A_{n} \omega_{n}=0
$$

with $\Omega=\omega_{n}+\epsilon \sigma_{1}$. Expanding this equation with $A_{n}=\frac{a}{2} \exp (j \Psi)$, and separating the resulting equation into real and imaginary parts, we obtain

$$
\begin{aligned}
& a^{\prime}=-\mu_{2_{1}} n^{4} a-\frac{n \xi_{2}}{\pi} \cos \Phi_{1}, \\
& a \Phi_{1}^{\prime}=\sigma_{1} a+\frac{n \xi_{2}}{\pi} \sin \Phi_{1}
\end{aligned}
$$

where $\Phi_{1}$ is given in equation (74). Following the same procedure as described in previous sections, the following frequency response equation is obtained:

$$
\left(n^{8} \mu_{2_{1}}^{2}+\sigma_{1}^{2}\right) a^{2}=\left(\frac{n \xi_{2}}{\pi}\right)^{2}
$$

Therefore, the solution of the flexural vibration is approximated by

$$
v(x, t)=\epsilon v_{1}(x, t)+O\left(\epsilon^{2}\right)=\epsilon\left[a \cos \left(\Omega T_{0}-\Phi\right)\right] \sin n \pi x+O\left(\epsilon^{2}\right)
$$

where $a$ and $\Phi$ are described by equations (99) and (100), respectively. The steady state value of the phase angle $\Phi$ is given by $\tan \Phi_{s}=\left(\frac{\sigma_{1}}{\mu_{21}}\right)$.

Equation (101) is the frequency response of the primary resonance. We note that it does not contain any nonlinearity, and hence corresponds to the frequency response condition for a linear oscillator. This result implies that the ordering relation $\xi=\epsilon^{2} \xi_{2}$ fails to capture the nonlinearity in the analysis.

In order to extend the analysis to include the nonlinearity, the length ratio $\xi$ is rescaled by letting $\xi=\epsilon^{3} \xi_{3}$. Moreover, the damping parameters $\mu_{2}, \mu_{3}$ and $\mu_{4}$ are rescaled according to equation (41). Expanding equations (25), (26) and (27) with these new ordering relations, and following the procedure described in Section 3.2, we reconsider the primary resonance. To simplify the procedure, only the essential results are presented in the following analysis; a complete description of the analysis can be found in [28]. The equation describing the first order flexural vibration is given in equation (78), and its solution at first order is given by equation (79). The elimination of secular terms in the second order equation requires that $\left(D_{1} A_{n}\right)=0$. This implies that the amplitude of the transverse response is independent of the time scale $T_{1}$. In removing the secular terms in the third order equation, it is determined that

$$
\begin{aligned}
& -2 j \omega_{n}\left(D_{1} A_{n}\right)+A_{n}(n \pi)^{2} n^{6}\left(\alpha_{1} \alpha_{2}+\alpha_{1} \alpha_{3}\right)-2 j \omega_{n} n^{4} \mu_{2_{2}} A_{n}-j(n \pi)^{4} \omega_{n} \mu_{t_{1}} A_{n}^{2} \bar{A}_{n} \\
& +2 j \frac{n^{4}}{\pi^{2}} \mu_{3_{2}} A_{n}-\left[\frac{15}{8}-\left(\frac{1}{3}+S\right)(n \pi)^{2}\right](n \pi)^{2} n^{4} A_{n}^{2} \bar{A}_{n}-j \frac{\xi_{3}}{n \pi} \exp \left(j \sigma_{2} T_{2}\right)=0
\end{aligned}
$$


must hold, where $\Omega=\omega_{n}+\epsilon^{2} \sigma_{2}$. Expanding this equation with $A_{n}=\frac{a}{2} \exp (j \Psi)$, and separating the resulting equation into real and imaginary parts, we obtain

$$
\begin{aligned}
& a^{\prime}=-n^{4} \mu_{2_{2}} a-\frac{1}{8}(n \pi)^{4} \mu_{4_{0}} \omega_{n} a^{3}+\frac{n^{2}}{\pi^{2}} \mu_{3_{2}} a-\frac{\xi_{3}}{n \pi} \cos \Phi_{2}, \\
& a \Phi_{2}^{\prime}=\sigma_{2} a-\Delta_{2} a^{3}+\Delta_{3} a+\frac{\xi_{3}}{n \pi} \Omega^{2} \sin \Phi_{2}
\end{aligned}
$$

where $\Phi_{2}$ is defined in equation (86), $\Delta_{2}$ is defined in equation (84) and $\Delta_{3}$ is defined in equation (85). After few manipulations, the following frequency response condition is obtained:

$$
\sigma_{2}=\left[\Delta_{2} a^{2}-\Delta_{3}\right] \pm \sqrt{\left(\frac{n \xi_{3}}{a \pi}\right)^{2}-\left[n^{4} \mu_{2_{2}}+\frac{1}{8}(n \pi)^{4} \mu_{4_{0}} \omega_{n} a^{2}-\frac{n^{2}}{\pi^{2}} \mu_{3_{2}}\right]^{2}}
$$

This equation indicates that the maximum steady-state response amplitude is given by

$$
\left(\frac{n \xi_{3}}{\pi}\right)^{2}-a_{p}^{2}\left[n^{4} \mu_{2_{2}}+\frac{1}{8}(n \pi)^{4} \mu_{4_{0}} \omega_{n} a_{p}^{2}-\frac{n^{2}}{\pi^{2}} \mu_{3_{2}}\right]^{2}=0
$$

and that it occurs at the frequency

$$
\Omega_{p}=\omega_{n}+\epsilon \sigma_{p}=\omega_{n}+\epsilon^{2}\left(\Delta_{2} a_{p}^{2}-\Delta_{3}\right)
$$

Since $\Delta_{2}<0$ and $\Delta_{3}>0$ for each individual mode, $\sigma_{p}<0$. Therefore, the frequency response curves bend to the left. Figure 5 provides an example for this frequency response equation, which shows the usual 'jump' behavior associated with a softening nonlinearity. From Figure 5, it is clear that when $\Omega$ is located between points $b$ and $c$, there exists more than one steady state response. Those steady state responses located along the $b c$ portion of the response curve are unstable, and hence will not be observed either in simulations or experiments. For frequencies between points $b$ and $c$ in Figure 5, the initial conditions will decide the steady state response. Saddle node bifurcations $[29,30]$ occur at the turning points $b$ and $c$ in Figure 5 .

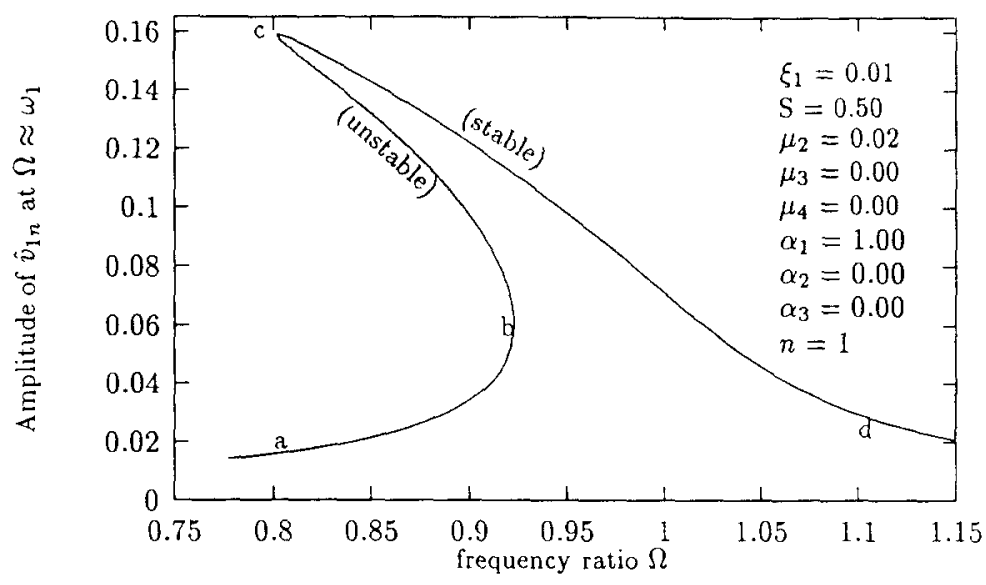

Fig. 5. Frequency response equation for the primary resonance. 
The solution of the transverse vibration of the connecting rod $v(x, t)$ can now be approximated by

$$
v(x, t)=\epsilon v_{1}(x, t)+O\left(\epsilon^{2}\right)=\epsilon\left[a \cos \left(\Omega T_{0}-\Phi\right)\right] \sin n \pi x+O\left(\epsilon^{2}\right)
$$

where $a$ and $\Phi$ are described in equations (104) and (105), respectively.

REMARKS. Before proceeding to the next section, there are a few points which should be made. First, we again compare the results from the linear and nonlinear analysis. By neglecting the effects of shear deformation, rotary inertia, and the friction parameters $\mu_{3_{2}}$ and $\mu_{4_{10}}$ in equations (104) and (105), we obtain

$$
\begin{aligned}
& a^{\prime}=-n^{4} \mu_{2_{2}} a-\frac{n \xi_{3}}{\pi} \cos \Phi_{2}, \\
& a \Phi_{2}^{\prime}=\sigma a+\Delta_{2} a^{3}+\frac{n \check{\xi}_{3}}{\pi} \sin \Phi .
\end{aligned}
$$

The resultant frequency response equation takes the form:

$$
a^{2}\left[\left(\sigma_{2}-\Delta_{2} a^{3}\right)^{2}+\left(n^{4} \mu_{22}\right)^{2}\right]=\left(\frac{n \xi_{3}}{\pi}\right)^{2} .
$$

Basically, the equations describing the variation of the amplitude of the response (equations (99) and (104)) are exactly the same. The only difference between linear and nonlinear analyses appears in the equations describing the variation of the phase angle (equations (100) and (105)). Moreover, the frequency response equations (101) and (112) coincide with each other in their linear parts. To show this, substitute $\mu_{2_{2}}=\epsilon \mu_{1_{1}}, \xi_{3}=\epsilon \xi_{2}$ and $\sigma_{2}=\epsilon \sigma_{1}$ into equation (112). Then equation (112) reduces to equation (101) as $a$ approaches zero. This implies that when the response amplitude is small, linear theory works well, as expected. However, as the response amplitude increases, the nonlinearity affects the system by changing the rate at which energy is put into the system. As a consequence, a new 'dynamic equilibrium' will be reached when the rate at which energy put into the system is balanced by the rate of energy dissipation.

It is clear that shear deformation and rotary inertia, included in $\Delta_{3}$, do not show up in the first order solution. They do affect the primary resonance by shifting to the left the entire backbone curve, which is locus of the peak amplitudes associated with the frequency response curves, an amount of $\Omega=\omega_{n}+O\left(\epsilon^{2}\right)$.

\subsection{Superharmonic Resonance $\left(2 \Omega \approx \omega_{n}\right)$}

In analyzing the particular solution of $\hat{v}_{2 n}$ in Section 3.1, it was assumed that the frequency ratio $\Omega$ was not near $2 \omega_{n}$ or $\frac{\omega_{n}}{2}$, and we then proceeded to analyze the equations of higher order. In the current section, the superharmonic resonance case in which the frequency ratio $\Omega$ is near $\frac{\omega_{n}}{2}$ is investigated. To consider this case, the frequency ratio $\Omega$ is expressed as $2 \Omega=\left(\omega_{n}+\epsilon \sigma_{1}\right)$. The damping parameter $\mu_{2}$ is rescaled as $\mu_{2}=\epsilon \mu_{21}$, and the parameter $\mu_{4}$ is rescaled as $\mu_{4}=\mu_{4_{0}}$. Equations (25), (26) and (27) are expanded with these ordering relations, and the procedure described in Section 3.2 is repeated. The first order flexural vibration associated with the connecting rod is described by equation (58), and the general solution of $\tilde{v}_{1 n}$ is given in equation 
(59). The elimination of the secular terms from the particular solution of $\hat{v}_{2 n}$ requires that

$$
\begin{aligned}
& -2 j \omega_{n}\left(D_{1} A_{n}\right)-2 j n^{4} \mu_{2_{1}} \omega_{n} A_{n}+\frac{\mu_{4_{0}}}{6} \xi_{1}^{2}(n \pi) \omega_{n} \exp \left(j \sigma_{1} T_{1}\right) \\
& +\left(\frac{\xi_{1}^{2}}{n \pi}\right)\left(\frac{\omega_{n}^{2}}{4}\right)\left[\frac{1-\cos n \pi}{2}-\frac{1}{6}(1+2 S)(n \pi)^{2}\right] \exp \left(j \sigma_{1} T_{0}-j \frac{\pi}{2}\right)=0 .
\end{aligned}
$$

Expanding this equation with $A_{n}=\frac{a}{2} \exp (j \Psi)$, and then separating the result into real and imaginary parts, yields the following equations:

$$
\begin{aligned}
& a^{\prime}=-n^{4} \mu_{2_{1}} a+\frac{\mu_{4_{0}}}{6}(n \pi) \xi_{1}^{2} \sin \Phi_{1}-\frac{\xi_{1}^{2} \omega_{n}}{24 n \pi}\left[3(1-\cos n \pi)-2 \frac{\Delta_{1}}{n^{2}}\right] \cos \Phi_{1}, \\
& a \Phi_{1}^{\prime}=\sigma_{1} a+\frac{\mu_{4_{0}}}{6}(n \pi) \xi_{1}^{2} \cos \Phi_{1}+\frac{\xi_{1}^{2} \omega_{n}}{24 n \pi}\left[3(1-\cos n \pi)-2 \frac{\Delta_{1}}{n^{2}}\right] \sin \Phi_{1}
\end{aligned}
$$

where $\Phi_{1}$ is given in equation (74), and $\Delta_{1}$ is defined by equation (75). After few manipulations, the following equation is obtained for the frequency response associated with this superharmonic resonance:

$$
a_{s}^{2}\left(n^{8} \mu_{2_{1}}^{2}+\sigma_{1}^{2}\right)=\xi_{1}^{4}\left[\frac{\mu_{4_{0}}}{6}(n \pi)\right]^{2}+\frac{\omega_{n}^{2}}{(24 n \pi)^{2}}\left[3(1-\cos n \pi)-2 \frac{\Delta_{1}}{n^{2}}\right]^{2} .
$$

Figure 6 provides an example for this frequency response. For $\Omega$ near $\frac{\omega_{2}}{2}$, the response of the flexural vibration can be approximated by

$$
v(x, t)=\epsilon v_{1}\left(x, T_{0}, T_{1}, T_{2}\right)+O\left(\epsilon^{2}\right)=\epsilon\left[\Lambda_{1} \sin \Omega T_{0}+a \cos \left(2 \Omega T_{0}-\Phi\right)\right] \sin n \pi x+O\left(\epsilon^{2}\right)
$$

where $\Lambda_{1}$ is given in equation (60), while $a$ and the phase angle $\Phi$ are described by equations (114) and (115), respectively.

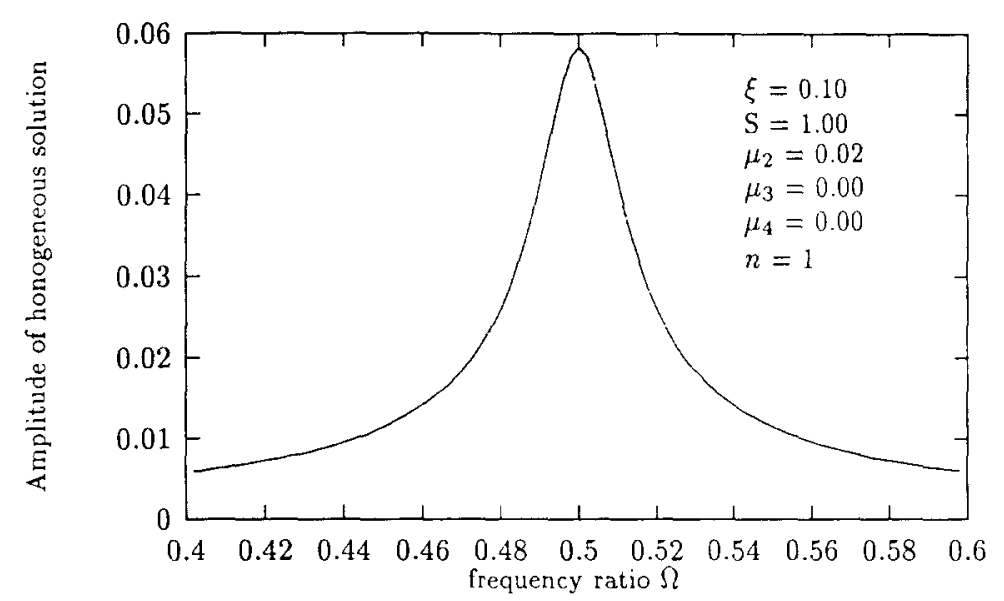

Fig. 6. Frequency response equation for the superharmonic resonance $\left(\Omega \approx \frac{\omega_{n}}{2}\right)$. 
REMARKS. Equation (117) is the first order approximate solution to the flexural vibration associated with the connecting rod near $\Omega=\frac{1}{2}$. Note that this solution consists of two parts, corresponding to the homogeneous and the particular solutions. A simple calculation of the eigenvalues associated with these steady state responses indicates that the homogeneous solution is stable for $\mu_{2_{1}}>0$. Hence, a stable steady state superharmonic resonance exists under quite general conditions and the response is the sum of two periodic functions. The particular solution is, of course, of frequency $\Omega$, while the homogeneous solution oscillates with frequency $2 \Omega$. The magnitude of the homogeneous part of the response is proportional to the square of the length ratio $\xi_{1}$, while the particular part of the response is proportional to $\xi_{1}$. This implies that the homogeneous solution vanishes more rapidly than the particular solution as the length ratio $\xi$ approaches zero. This result is expected, since due to the kinematic constraints, higher harmonics becomes more prominent for larger length ratios.

This resonance arises from the $2 \Omega$ harmonics of the external and parametric excitation terms, and nonlinear effects are not included in the analysis at this order. To include the nonlinear effects, it is necessary to carry the analysis to higher order.

\section{Discussion}

In this section, the results from Section 3 are summarized and the effects of the length ratio $\xi$, the frequency ratio $\Omega$, the mass ratio $S$, and the damping parameters $\mu_{2}, \mu_{3}$ and $\mu_{4}$ on each resonance are discussed in turn.

\subsection{Effect of the Length Ratio $\xi$}

As was mentioned in Section 1, an increase of the inertial force is primarily responsible for the failure of the traditional, linear dynamic analysis. In this problem, the acceleration components $a_{x}$ and $a_{y}$ and, consequently, the inertial forces, are proportional to the length ratio $\xi$ (see equations (3) and (4)). The general solution of the first order linear flexural vibration problem is given as a combination of the particular and homogeneous solutions. The magnitude of the particular

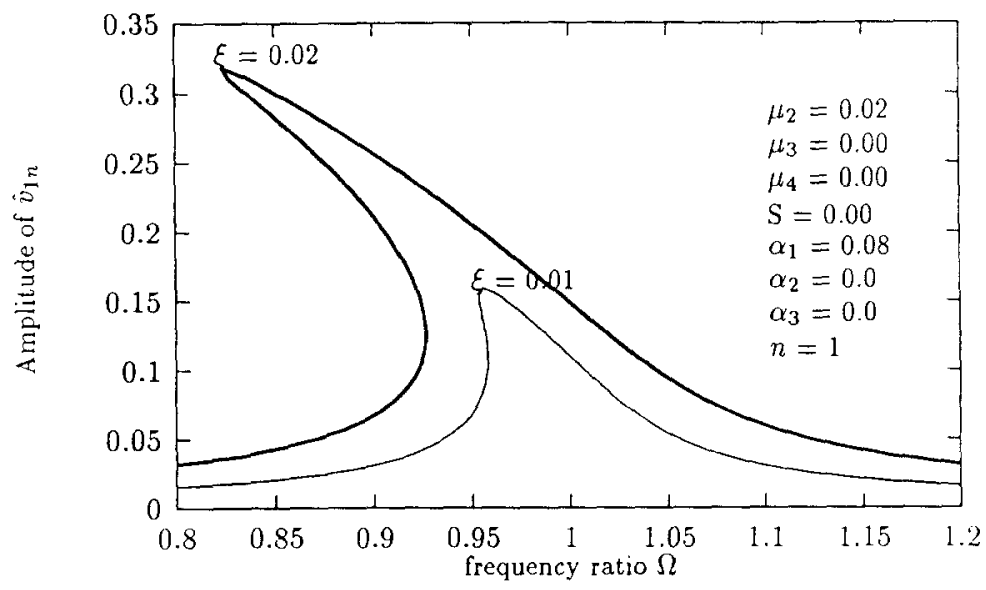

Fig. 7. Influence of $\xi$ on the primary resonance. 


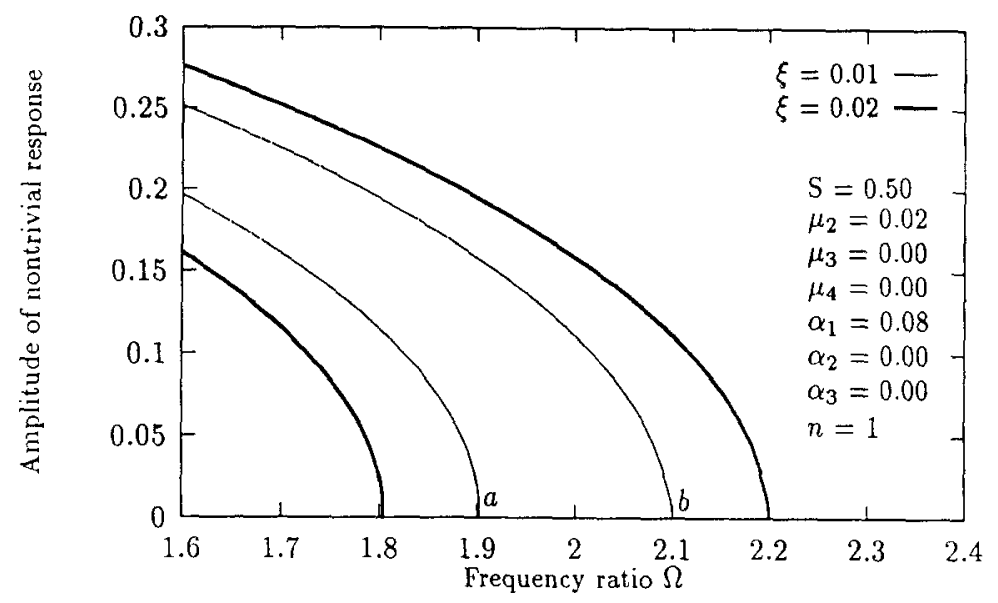

Fig. 8. Influence of $\xi$ on the principal parametric resonance.

solution is proportional to $\xi$. If no resonance occurs, the free oscillation term will decay to zero, and hence the steady state response contains only the particular solution. Therefore, the amplitude of the response will be proportional to the length ratio for nonresonant cases. In the case of the primary resonance, the peak amplitude of the resonance is proportional to $\xi$ (equation (107)). Figure 7 shows the effect of $\xi$ on the primary resonance. The frequency at which this peak amplitude occurs depends upon the square of the magnitude of the particular solution, i.e., is proportional to $\xi^{2}$ (equation (108)).

In the case of the principal parametric resonance, the width of the frequency region in which the trivial response becomes unstable is also proportional to $\xi$ (equation (77)). Figure 8 shows the effect of the length ratio on the main nose of instability. We note that, in Figure 8 , the width between the transition curves increases as $\xi$ increases. Figure 8 also shows the effect of the length ratio on the amplitude of the nontrivial responses. It is seen that, as expected, the amplitude of the stable non-trivial branch (emanating from point $b$ ) increases as $\xi$ is increased.

In the case of the $k^{\text {th }}$ order superharmonic resonance, the magnitude of the non-zero homogeneous solution is proportional to $\xi^{k}$. Hence, the $k \omega_{n}$ harmonic of the response will become more prominent for large values of $\xi$. This can be predicted from linear theory, but quantitative results require the use of a method such as the one employed herein.

\subsection{Effect of the Mass Ratio $S$}

The effect of this parameter is not immediately clear. Generally speaking, it acts as a primary source of nonlinearity in the system. In the case of primary resonance, a slight increase of $S$ will cause a significant increase of the value of $\sigma_{p}$, the value of detuning at which the peak amplitude occurs. To make this point more clear, consider the following cases: $S=0$ and $S=1$. The frequency corresponding to the peak amplitude of the primary resonance is shifted to the left by an amount $\epsilon^{2} \sigma_{p}$. When there is no mass attached to the slider end, the value of $\sigma_{p}$ is given by

$$
\left(\sigma_{p}\right)_{S=0}=\left(\frac{\pi^{2}}{3}-\frac{15}{8}\right) \frac{\pi^{2}}{8}
$$




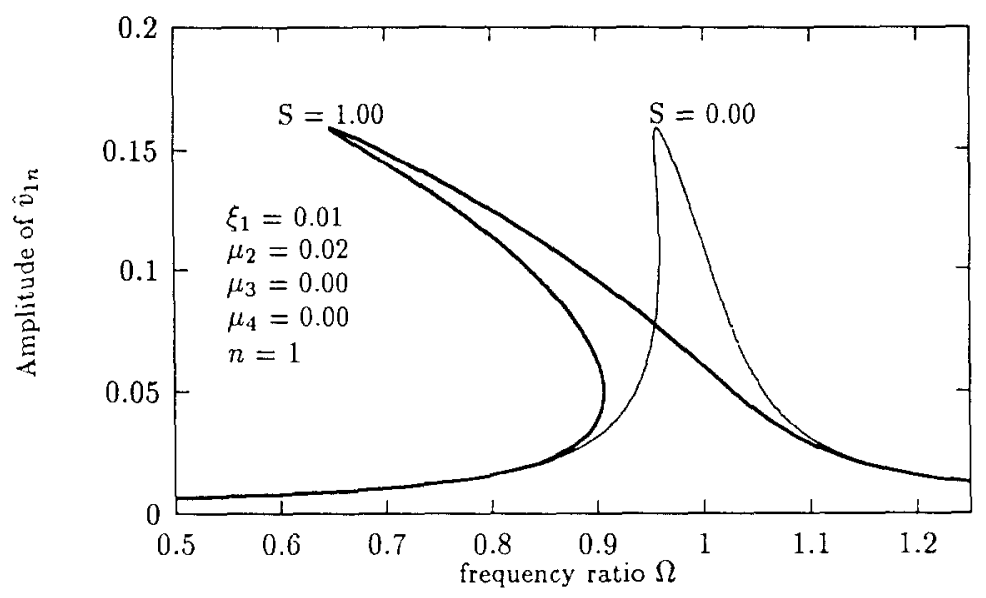

Fig. 9. Influence of $S$ on the primary resonance.

for the first mode $(n=1)$. When $S=1$, the value of $\sigma_{p}$ becomes

$$
\left(\sigma_{p}\right)_{S=1}=\left(\frac{\pi^{2}}{3}-\frac{15}{8}\right) \frac{\pi^{2}}{8}-\frac{\pi^{4}}{8}=\left(\alpha_{p}\right)_{S=0}-\frac{\pi^{4}}{8} .
$$

A rough estimate of the difference between these two values of $\sigma_{p}$ shows that $\left(\sigma_{p}\right)_{S=1}$ is about eight times the value of $\left(\sigma_{p}\right)_{S=0}$. Moreover, this ratio will increase as the value of $S$ increases beyond 1. This implies that the value of $\sigma_{p}$ is very sensitive to the value of $S$. Figure 9 demonstrates this influence of the mass ratio $S$ on the primary resonance.

The mass ratio $S$ affects the principal parametric resonance by increasing the width of the main noise of instability, as shown in Figure 10. Figure 11 shows the amplitude of the nontrivial homogeneous response associated with the principal parametric resonance. It is interesting to note that, although the instability region is widened by increasing $S$, the amplitude of the stable, nontrivial branch is decreased. The physical reason for these features is the following: The slider mass provides a periodic inertial end load which helps destabilize the system in the usual way when $\Omega$ is near 2 . However, once unstable, the motion is then limited by the same inertial

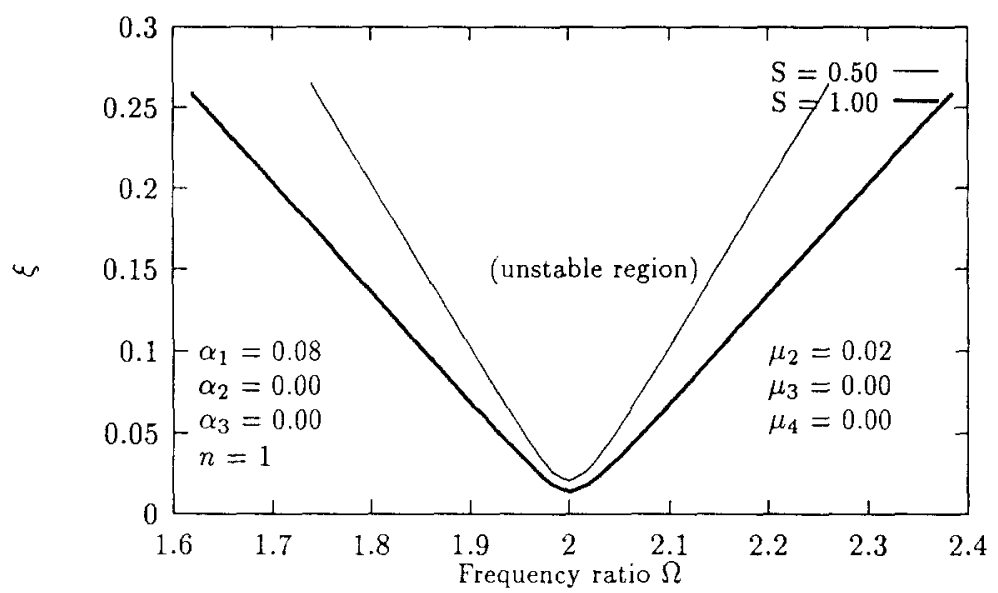

Fig. 10. Influence of $S$ on the main nose of instability. 


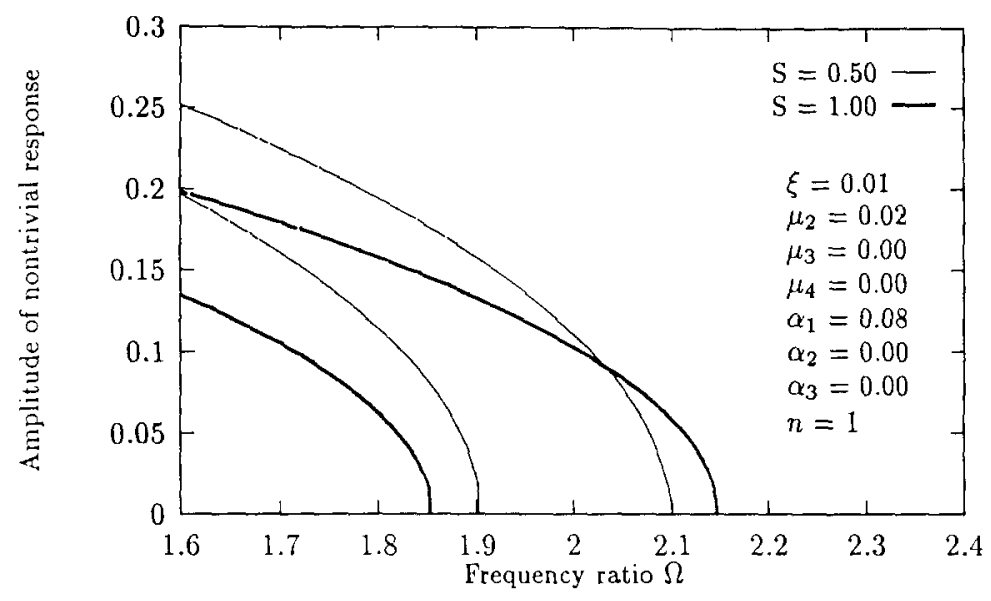

Fig. 11. Influence of $S$ on the principal parametric resonance.

resistance through the nonlinear foreshortening effects of bending. The slider friction plays a similar role, as described in Section 4.3.

In the superharmonic resonance, $S$ has a very interesting effect on the response. In this case, the component of the steady-state response with frequency $2 \Omega$ has an amplitude of $a_{s}$, given in equation (116). The left hand side of equation (116) is independent of $S$, so that the dependence of $a_{s}$ on $S$ can be obtained by considering the right hand side of equation (116). Based on this, we find that $a_{s}$ reaches a minimum with $2 \Delta_{1}-3 n^{2}(1-\cos n \pi)=0$. Figure 12 shows the dependence of $a_{s}$ on $S$ for the first mode. Note that $a_{s}$ decreases as $S$ increases from zero to about 0.11 , and then $a_{s}$ increases as $S$ increases beyond 0.11 . This implies that a proper choice of the mass ratio can help in suppressing the amplitude associated with the $2 \Omega$ component of the response near this superharmonic resonance.

\subsection{Effect of Damping Parameters $\mu_{2}, \mu_{3}$ and $\mu_{4}$}

The internal material damping $\mu_{2}$ has a favorable effect on the overall response of the system. In

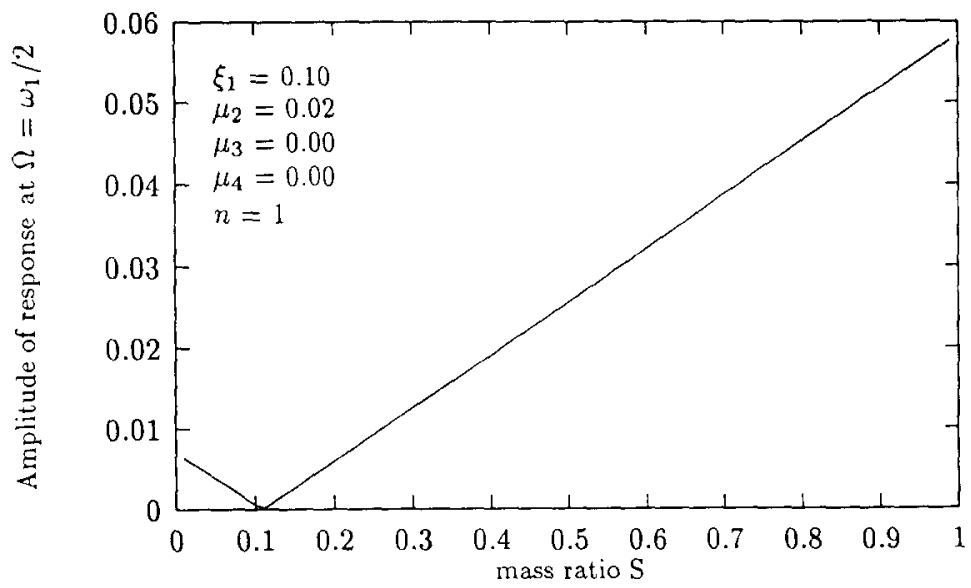

Fig. 12. Influence of mass ratio $S$ on the amplitude of superharmonic resonance $\left(\Omega \approx \frac{\omega_{n}}{2}\right)$. 


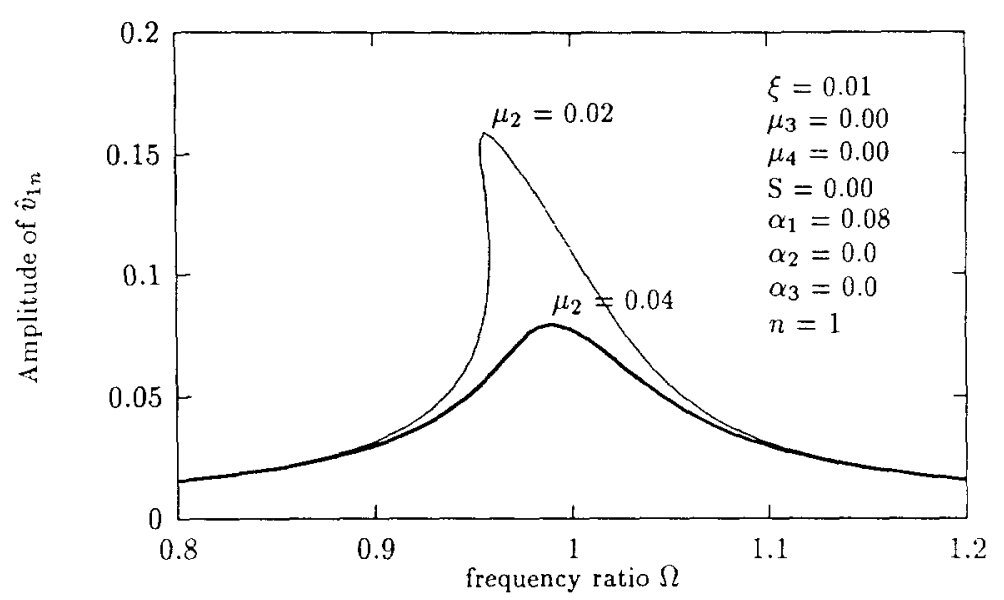

Fig. 13. Influence of $\mu_{2}$ on the primary resonance.

the primary resonance case, the presence of $\mu_{2}$ in the frequency response equations (101) and (112) reduces the peak amplitude of the system, as indicated in Figure 13. In the principal parametric resonance, the width of the main nose of instability decreases as the value of $\mu_{2,}$ is increased; see equation (76). Moreover, the amplitude of the response decreases as the value of $\mu_{2,}$ is increased; see equation (87). The small change in $\mu_{2}$ used in Figure 13 yields a very slight reduction in the width of the unstable region and in the amplitude of the nontrivial response branches. For large increase in $\mu_{2}$, the width of the main nose of instability reduces to zero for a specific value of $\xi$. In the superharmonic resonance, the amplitude of the free oscillation term decreases as the value of $\mu_{2,}$ is increased (equations (116)). These observations show that the presence of $\mu_{2}$ has a favorable effect on the response for all the resonances considered here.

The bearing friction $\mu_{3}$ has an adverse effect on the overall response of the system. This can be shown by considering the negative sign associated with parameter $\mu_{3}$ in the frequency response equation of each resonance case. The presence of this parameter implies a constant positive energy flux from bearing friction, which in turn implies an increase in the amplitude of the response. The reason for this is that the relative angular motion associated with each joint (points $\mathbf{A}$ and $\mathbf{B}$ ) is constant at first order. The presence of $\mu_{3}$ will thus cause a constant frictional moment acting on each joint. and these friction moments enlarge the transverse deformation. Figure 14

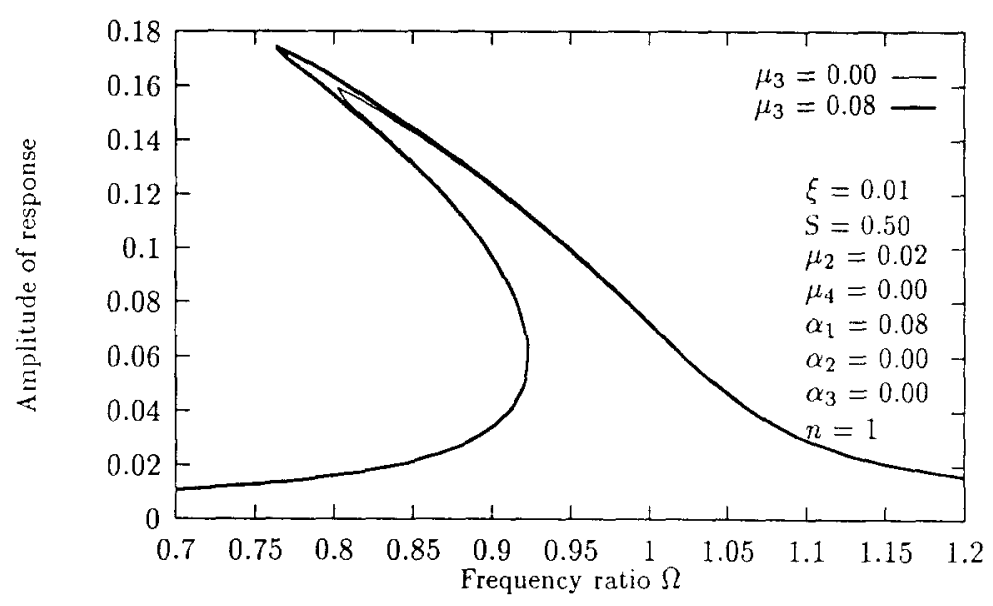

Fig. 14. Influence of $\mu_{3}$ on the primary resonance. 
shows the influence of $\mu_{3}$ on the primary resonance. The same increase in $\mu_{3}$ has no perceptible effect on the principal parametric resonance. From these two cases, it is seen that the influence of $\mu_{3}$ on each resonance case is not very significant, although the effect is negative.

The effect of the friction parameter $\mu_{4}$, which is used to model the friction between the slider mass and its contact surface, is complicated. In order to have a clear picture regarding the effect of $\mu_{4}$ on the response, it is useful to consider the nature of this friction force. The slider friction force is composed of the actions of the rigid body motion as well as the elastic deformation. When the response amplitude is small, slider friction is mainly composed of the friction introduced by rigid body motion. As the value of $\mu_{4}$ increases, slider friction increases. This results in an increase in the axial force acting on the connecting rod and, consequently, the response amplitude increases and the possibility for parametric resonances is enhanced. From this consideration, it appears that $\mu_{4}$ has an adverse effect on the response. This can be observed in the second order superharmonic $\left(\Omega \approx \frac{1}{2}\right)$ resonance (equation (116)). Moreover, the width of the main nose of instability associated with the principal parametric resonance increases slightly as the value of $\mu_{4}$ is increased (equations (93) and (94)). However, an increase of the response amplitude is accompanied by an increase in the foreshortening induced by the transverse displacement $v(x, t)$ of the connecting rod. The component of the friction force associated with this deformation acts as a resistance to further deformation, and hence it also has a favorable effect. The net effect of these competing effects can be observed in the principal parametric resonance, as shown in Figure 15. From this figure, it is seen that $\mu_{4}$ has a favorable effect on the response amplitude, while it also has a slight tendency to increase the width of the main nose of instability. Figure 16 shows that the friction parameter $\mu_{4}$ has a favorable effect on the primary resonance. This is caused by the friction force introduced by large elastic deformation, and no parametric instability occurs near primary resonance.

\subsection{Effect of Shear Deformation and Rotary Inertia}

In this work, the effects of shear deformation and rotary inertia are included in the parameter $\Delta_{3}$ (equation (85)). Their effects do not appear in the first order solution and, in all resonance cases, only appear in a higher order approximation. In the primary resonance, the presence of shear deformation and rotary inertia affect the frequency response curve in such a way that the locus of

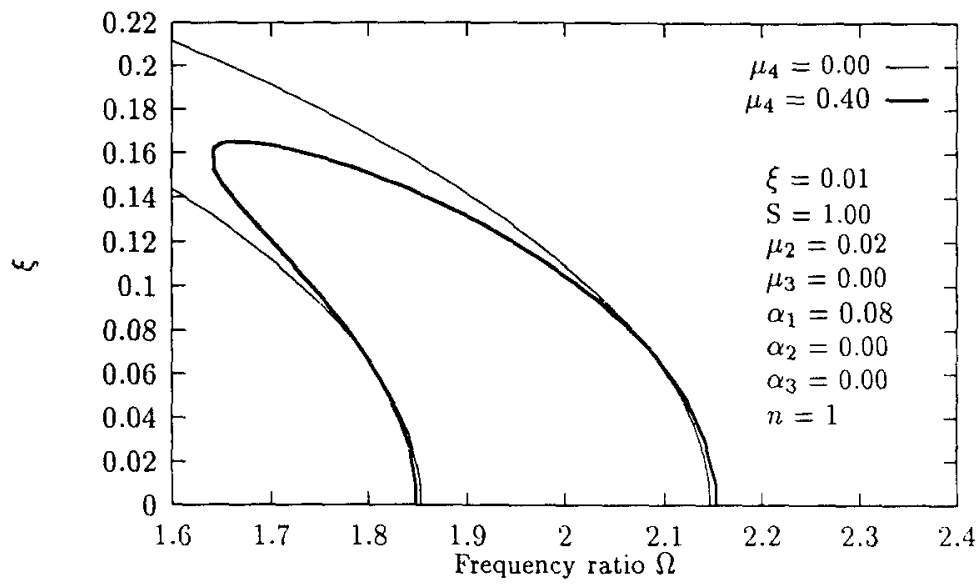

Fig. 15. Influence of $\mu_{4}$ on the principal parametric resonance $\left(\Omega \approx 2 \omega_{1 n}\right)$. 


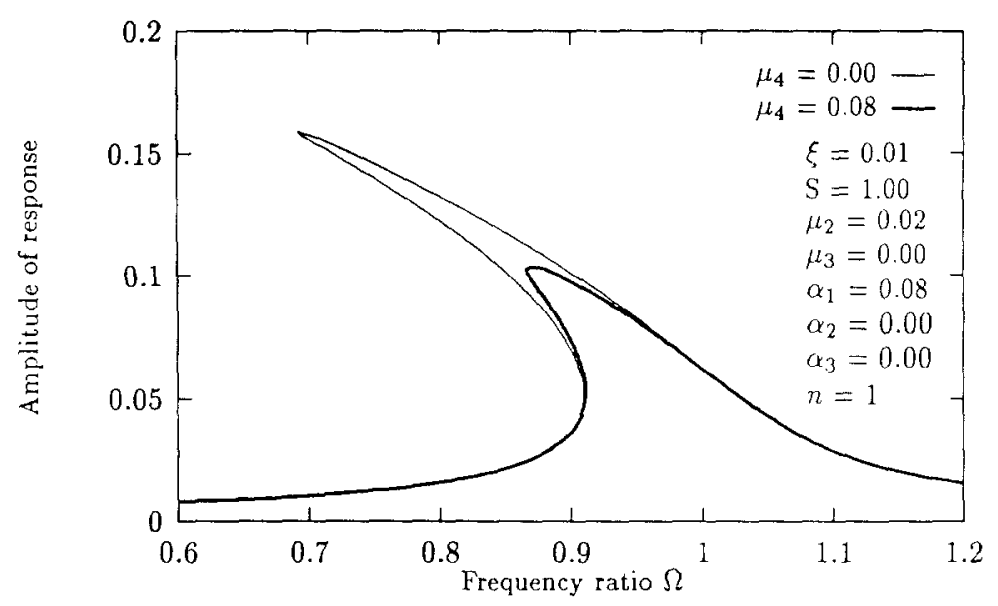

Fig. 16. Influence of $\mu_{4}$ on primary resonance $\left(\Omega \approx \omega_{1 n}\right)$.

the peak amplitudes, i.e., the backbone curve, does not originate from $\omega_{n}$. The entire backbone curve, including its zero amplitude origin, is shifted to the left by a constant amount of $O\left(\epsilon^{2}\right)$ which is independent of the amplitude of the response. In the principal parametric resonance, the $\Delta_{3}$ term causes the center of the region in which the trivial solution becomes unstable to shift to the left by an amount of $O\left(\epsilon^{2}\right)$, while the width of this region remains unchanged. The effects of shear deformation and rotary inertia are very small and hence the profiles associated with these two resonances are almost unchanged where $\Delta_{3}$ is neglected. Shear deformation and rotary inertia do not affect the superharmonic resonance in which $\Omega$ is near $\frac{\omega_{n}}{2}$.

\section{Conclusions}

In this paper, the flexural vibration response associated with the elastic connecting rod of an otherwise rigid slider crank mechanism has been investigated by using the method of multiple scales. Similar perturbation methods, such as the regular expansion method $[12,15]$ and the method of averaging [10,11], have been applied to study mechanism system. However, all previous investigations have provided only linear results. In particular, analyses of the stability associated with the steady state response and detailed studies of the nonlinear resonances have not been carried out.

Two different models have been used to study this problem. The dynamic behavior of the connecting rod described by a single nonlinear ordinary differential equation, obtained by direct modal truncation of the partial differential equations, is considered in [20]. This procedure follows the works by Viscomi and Ayre [4] and Badlani [15], although the effects of bearing and slider friction are included in [28]. Extensive simulations results are given in [20] which verify the validity of the approximate solutions for $\xi<0.1$. In the model presented in this paper, the dynamic response of the elastic connecting rod is described by a set of partial differential equations. The following conclusions are drawn by comparing the present results with those in [20]: (1) these models provide the same results in the linear part, (2) they also provide the same qualitative results in the nonlinear part, and (3) the distributed parameter model provides a stronger nonlinearity than lumped parameter models. Moreover, the mass ratio $S$ is the only 
source of the nonlinearity in the lumped parameter model, while for the distributed parameter model, finite deformation effects provide a source of nonlinearity in addition to the mass ratio $S$. A detailed discussion of this comparison is given in [28].

The results presented in this investigation represent a successful extension of previous efforts in that the effects of nonlinearities have been analyzed in a systematic manner. The major drawback of this approach is that tedious manipulations are involved in analyzing the higher order resonances and the results are useful only over a limited parameter range, in particular, $\xi$ must be small. Also, the analysis of a mechanism with two or more flexible elements becomes unreasonably complicated for such a treatment.

With the advent of high speed computers, the finite element approach becomes more and more favorable for specific design purposes. However, like most numerically based approaches, the finite element approach can only provide pointwise information in parameter space. A recent finite element study [31] used more than one hundred simulations with a commercially available package to obtain a single response curve. In contrast, an analytical approach provides important information regarding the influence of various system parameters on the response, and furnishes theoretical explanations for some of the nonlinear phenomena associated with these systems.

Future work in this area can be separated into several distinct directions. This analysis can be directly extended to study the elastic behavior associated with the flexible coupler of an otherwise rigid four-bar mechanism. Moreover, the effects of off-set, an applied force on the slider mass, and out-of-plane deformation can be included. A detailed study of the slider crank mechanism with low frequency ratio $\Omega$ and large length ratio $\xi$ is also recommended, since this is a parameter range which may be amenable to asymptotic methods and is important in applications. Moreover, the inclusion of multimode solutions and intermodal coupling should be considered in a nonlinear analysis. The phenomenon of internal resonance [26] between modes can be examined in this context. Systematic experimental studies, such as the recent work of Beale et al. [19], should also be carried out.

As for the general improvement in the analysis techniques for elastic mechanism problems, a more thorough understanding of the inertial force is required. In all previous analytical works, the dynamics of the flexible link is formulated relative to a coordinate system that follows the rigid body motion of the beam. This approach presumes an infinitesimal strain and is sometimes referred to as the shadow beam approach. For large overall motion and strains, geometrically exact formulations should be employed. Recent results from Simo and Vu-Quoc [32,33] may prove useful for this extension.

\section{Acknowledgements}

This work was supported by NSF grant MSM-8915453, and was carried out while both authors were at Michigan State University. The authors are grateful to Dr. Alan Haddow of Michigan State University for providing many helpful comments during the course of this work.

\section{References}

1. Chu, S. and Pan, K. C., 'Dynamic responses of a high-speed slider-crank mechanism with an elastic connecting rod', ASME Journal of Engineering for Industry 97, 1975, 542-550. 
2. Golebiewski, E. P. and Sadler, J. P., Analytical and experimental investigation of elastic slider-crank mechanisms', ASME Journal of Engineering for Industry 93, 1976, 1266-1271.

3. Sutherland, G. H., 'Analytical and experimental investigation of a high-speed elastic-member linkage', ASME Journal of Engineering for Industry 98, 1976, 788-794.

4. Viscomi, B. V. and Ayre, R. S., 'Nonlinear dynamics response of elastic slider crank mechanism', ASME Journal of' Engineering for Industry, 93, 1917, 251-262,

5. Erdman, A. G. and Sandor, G. N. 'Kineto-elastodynamics-a review of the state of the art trends', Mechanism and Machine Theory 7, 1972, 19-33.

6. Lowen, G. G. and Jandrasits, W. G.. 'Survey of investigation into the dynamic behavior of mechanisms containing links with distributed mass and elasticity'. Mechanism and Machine Theory 7, 1972, 3-17.

7. Lowen, G. G. and Chassapis, C., 'The elastic behavior of linkages: An update', Mechanism and Machine Theory 21. 1986, 33-42.

8. Thompson, B. S. and Sung. C. K., 'A survey of finite element techniques for mechanism design', Mechanism and Machine Theory 21, 1986, 351-359.

9. Neubauer, A. H. Jr., Cohen, R., and Hall, A. S. Jr., 'An analytical study of the dynamics of an elastic linkage', ASME Journal of Engineering for Industry 88, 1966, 311-317.

10. Jasinski, P. W., Lee, H. C., and Sandor, G. N., 'Stability and steady-state vibrations in a high-speed slider-crank mechanism', Journal of Applied Mechanics 37, 1970, 1069-1076.

11. Jasinski, P. W., Lee, H. C., and Sandor, G. N., 'Vibration of elastic connecting rod of a high-speed slider-crank mechanism, ASME Journal of Engineering for Industry 93, 1971, 636-644.

12. Badlani, M. and Kleinhenz, W., 'Dynamic stability of elastic mechanisms', ASME Journal of Mechanical Design 101, 1979, 149-153.

13. Tadjbakhsh, I. G., 'Stability of motion of elastic planar linkage with application to slider-crank mechanism", $A S M E$ Journal of Mechanical Design 104, 1982. 698-703.

14. Zhu, Z. G. and Chen, Y., 'The stability of the motion of a connecting rod', ASME Journal of Mechanisms, Transmission, and Automation Design 105, 1983, 637-640.

15. Badlani, M. and Midha, A., 'Effect of internal material damping on the dynamics of a slider-crank mechanism', ASME Journal of Mechanisms, Transmission, and Automation Design 105, 1983, 452-459.

16. Farhang, K. and Midha, A.. 'Investigation of parametric vibration stability in slider-crank mechanism with elastic coupler', Proceeding of 13th ASME Conference on Mechanical Vibration and Noise De-Vol. 37. Miami, FL, October 1991, 167-176.

17. Beale, D. and Lee, S.-W.. 'Investigation of parametric resonance stability in a flexible rod slider crank mechanism', Proceeding of 13th ASME Conference on Mechanical Vibration and Noise De-Vol. 37. Miami. FL, October 1991, 161-166.

18. Beale, D. G. and Lee, S.-W., 'Steady state response of a slider crank with flexible rod'. Nonlinear Dynamics (to appear).

19. Beale, D. and Halbig, D., 'Experimental high speed response of a slider crank', in Fourth Conference on Nonlinear Vibration, Stability, and Dynamics of Structures and Mechanisms, Blacksburg, VA. June 7-11, 1992.

20. Hsieh, S.-R. and Shaw, S. W. 'Dynamic stability and nonlinear resonance of a flexible connecting rod: Single mode model', Journal of Sound and Vibration (to appear).

21. Ryan, R. R., 'Flexibility modeling methods in multibody dynamics, Ph.D. Thesis, Stanford University, 1986.

22. Yoo, Hong Hee, 'Dynamic modeling of flexible bodies in multibody systems', Ph.D. Thesis, University of Michigan, 1988.

23. Greenwood, D. T., Principles of Dynamics, Prentice-Hall, Inc, Englewood Cliffs, NJ, 1969

24. Housner, H., Applied Mechanics Dynamics, Van Nostrand, Princeton, NJ, 1959.

25. Timoshenko, S., Vibration Problem in Engineering, Van Nostrand, Princeton, NJ, 1990.

26. Nayfeh, A. H. and Mook, D. T., Nonlinear Oscillations, Wiley Interscience. New York, NY, 1979.

27. Dym, C. L. and Shames, 1. H., Solid Mechanics, A Variational Approach. McGraw-Hill, New York, NY, 1973.

28. Hsieh, S.-R., 'Nonlinear vibrations of a flexible connecting rod', Ph.D. Thesis, Michigan State University, 1991.

29. Wiggins, S., Introduction to Applied Nonlinear Dynamical Systems and Chaos, Springer-Verlag, New York. NY, 1990.

30. Guckenheimer, J. and Holmes, P.. Nonlinear Oscillations, Dynamical Systems, and Bifurcations of Vector Fields, Springer-Verlag, New York, NY, 1983.

31. Cronin, D. L. and Liu, H., 'Finite element analysis of the steady-state behavior of flexible mechanisms', in The First National Applied Mechanisms and Robotics Conference, Cincinnati, $\mathrm{OH}$, November 1989.

32. Simo, J. C. and Vu-Quoc, L., 'On the dynamic of flexible beam under large overall motions - the plane case: Part I', ASME Journal of Applied Mechanics 53, 1986, 849-854.

33. Simo, J. C. and Vu-Quoc, L., 'On the dynamic of flexible beam under large overall motions-the plane case: Part II', ASME Journal of Applied Mechanics 53, 1986, 855-863. 